\title{
A!
}

This is an electronic reprint of the original article.

This reprint may differ from the original in pagination and typographic detail.

Chi, Chang Koo; Murto, Pauli; Välimäki, Juuso

\section{All-pay auctions with affiliated binary signals}

Published in:

Journal of Economic Theory

DOI:

10.1016/j.jet.2018.10.010

Published: 01/01/2019

Document Version

Peer reviewed version

Published under the following license:

CC BY-NC-ND

Please cite the original version:

Chi, C. K., Murto, P., \& Välimäki, J. (2019). All-pay auctions with affiliated binary signals. Journal of Economic Theory, 179, 99-130. https://doi.org/10.1016/j.jet.2018.10.010

This material is protected by copyright and other intellectual property rights, and duplication or sale of all or part of any of the repository collections is not permitted, except that material may be duplicated by you for your research use or educational purposes in electronic or print form. You must obtain permission for any other use. Electronic or print copies may not be offered, whether for sale or otherwise to anyone who is not an authorised user. 


\title{
All-PAy Auctions With AfFiliated BinARy Signals
}

\author{
Chang Koo Chi ${ }^{1}$, Pauli Murto ${ }^{2}$, and Juuso Välimäki ${ }^{* 2}$ \\ ${ }^{1}$ Department of Economics, Norwegian School of Economics \\ ${ }^{2}$ Department of Economics, Aalto University School of Business
}

October 17, 2018

\begin{abstract}
We analyze all-pay auctions with affiliated values and binary signals. We analyze the unique symmetric equilibrium with any number of bidders and show that the bidders earn positive rents only if the equilibrium is monotone. We also characterize the symmetric equilibrium of the closely related two-player war of attrition.

We compare expected revenues across these formats. All-pay auctions result in lower expected rents to the bidders than standard auctions, but they also induce inefficient allocations in some models with affiliated private values. With two bidders, the effect on rent extraction dominates, and the all-pay auctions outperforms standard auctions in terms of expected revenue. With many bidders, standard auctions may result in higher expected revenue. The war of attrition outperforms the standard auctions in terms of revenue, but its ranking relative to all-pay auctions is ambiguous.
\end{abstract}

JEL CLASSIFICATION: D44, D82

KEYWORDS: All-pay auctions, war of attrition, common values, affiliated signals

\section{Introduction}

Competition for a scarce resource often takes the form of a contest. In nature, males of animal species expend energy and risk injury when competing for females and trees grow in height to compete for solar energy. Closer to economics, lobbyists spend money and effort in order to secure a decision that is favorable to their cause. Architectural competitions and online contests invite participants to work on a design problem or an algorithm, and the best result is awarded a prize. The key distinguishing feature of these examples is that the contestants have to decide how much effort to sink into the contest and the outcome is determined as a function of these efforts.

\footnotetext{
*Chang Koo Chi: chang-koo.chi@nhh.no; Pauli Murto: pauli.murto@aalto.fi; Juuso Välimäki: juuso.valimakidaalto.fi. We would like to thank Christian Krestel, Stephan Lauermann, the editor Xavier Vives, three referees, and numerous seminar audiences for helpful comments on the paper.
} 
Contest models are closely related to auctions. The simplest contest model is called an all-pay auction, where the prize is awarded to the contestant with the highest effort. The effort choice corresponds to the choice of a bid in an auction and the sunk cost nature of effort choice is emphasized by the requirement that all the bidders pay their bids. In terms of this analogy, a first price auction would be a contest with a promised effort level when winning. Just as for ordinary auctions, it is natural to consider contest models where the subjective estimates of the value of the prize are private information to the contestants. And just like in the case of auctions, it makes sense to ask how contests with correlated values differ from contests with independent values. Such informational linkages have been analyzed extensively in the auction literature, but have received much less attention in the contest literature.

In a two-bidder model, Rentschler and Turocy (2016) show that correlation makes it harder to assess a priori what kinds of signals on the quality of the prize are advantageous to any given bidder. We put more structure on our model, and we assume throughout that signals are affiliated, i.e. they co-move positively. This means that a contestant (or bidder) with a high signal expects more opponents with high signals than a bidder with a low signal. When winning, the prize is on average more valuable for a high signal bidder. But affiliation also implies that if the other contestants with high signals expend more effort, then the competition that a high signal bidder expects is also tougher. These effects are present for standard first price and second price auctions too, but a crucial difference between the formats is that in contrast to the standard formats, bids in the all-pay auction are sunk. This makes the increased competition much more harmful to bidders.

From an analytical point of view, the key implication of this enhanced competition effect relative to standard formats is that best-response bids or effort choices need no longer be monotonic in the signal. As is well understood, this may preclude the existence of an equilibrium in monotonic strategies, which may explain why the literature on affiliated all-pay auctions and contests is so scarce. ${ }^{1}$ When monotonicity is lost, equilibrium characterization becomes very difficult. To circumvent the issue of intractability, the previous literature assumes monotonicity of equilibria by a parameter restriction (e.g, Krishna and Morgan (1997) and Siegel (2014)) or provides algorithmic or partial characterizations of equilibrium in case of two bidders (e.g, Rentschler and Turocy (2016) and Lu and Parreiras (2017)). ${ }^{2}$ As we show in this paper, the conditions for monotonicity are quite restrictive and the existing characterizations do not allow for easy results on the qualitative features of the equilibria when monotonicity fails.

Our goal here is to provide a complete characterization of both monotone and non-monotone symmetric equilibria of the all-pay auction with an arbitrary number of symmetric players. For this purpose, we must use a simpler informational model. Rentschler and Turocy (2016) adopt

\footnotetext{
${ }^{1}$ The idea that affiliated signals coupled with costly bidding lead to a non-monotonic equilibrium dates back to Milgrom and Weber (1982), where they construct an example showing that a monotone equilibrium does not exist in case of costly participation. Krishna and Morgan (1997) demonstrate that all-pay auctions may not have a monotone equilibrium when affiliation is strong. See also Landsberger (2007).

${ }^{2}$ Appendix B of Lu and Parreiras (2017) provides one example that shows how to construct a non-monotone equilibria under a parametric (quadratic) restriction on bidders' valuation.
} 
a discrete signal space and they provide an algorithm for computing symmetric equilibria of a two-player all-pay auction for both the monotone and non-monotone case. We further simplify their model by assuming binary signals that are affiliated. Since we cover the case of an arbitrary number $N$ of bidders, we find it convenient to assume that the signals are also affiliated with another random variable that we call the state of the world. In the mineral rights model, this common variable can be taken to be the true value of the prize whereas in the case with affiliated private values, it is best thought of as a parameter determining the true distribution of bidder types in the population. Within this formulation, we can analyze the comparative statics of the model in the number of bidders while fixing the signal structure of an individual bidder.

The binary information structure is also ideal for isolating key economic forces that the correlated information brings to contest models. The key insight is the possibility of inefficient allocations (for private values models). We analyze in detail how this is influenced by the correlation structure and the number of contestants. We know from Rentschler and Turocy (2016) that such inefficiencies prevail in a general model. However, their model with a general signal space turns out to be very difficult to extend beyond two bidders, and is even hard to characterize in a systematic way when there are only two bidders.

We provide a full characterization of the symmetric equilibria for our game with any number of bidders. We start by showing the existence and uniqueness of symmetric equilibrium, and prove that the unique symmetric equilibrium takes one of two possible forms: either it is monotone in the sense that higher value bidders win with probability one against lower value bidders. Alternatively it is non-monotone: a bidder with a lower value wins with a positive probability against a higher value competitor. In the latter case, we show that no bidder receives a positive expected payoff in the game, i.e. all rents are fully dissipated. In monotone equilibria, players with high valuations do receive an information rent.

As long as our equilibria are monotone, the results just confirm what Krishna and Morgan (1997) find in their model. The main contribution of our paper lies in the analysis of the nonmonotone equilibrium. We show that the unique symmetric equilibrium is in non-monotone strategies for a large set of parameter values. In the mineral rights model, the symmetric equilibrium is always non-monotone if the number of contestants or bidders is large enough. In the affiliated private values model, the equilibrium tends to be non-monotone if the differences in the private values are not too large and as long as the affiliation is not too small. With independent private values, however, the equilibrium is always in monotone strategies since the strength of competition is independent of a contestant's own type.

The all-pay auction can be thought of as a static contest model in the sense that the bidders expend irreversible efforts or outlays before knowing who wins. In order to check the robustness of our insights, we also analyze a closely related dynamic contest model, the war of attrition. In that model, the contestants observe each other expending effort gradually up to the point where the last contestant remaining is declared the winner. Since such a model does not have a symmetric equilibrium for more than two players, we restrict to the two-player war of attrition, which is 
equivalent to a second-price all-pay auction. ${ }^{3}$ We show that the unique symmetric equilibrium of this game can also be in non-monotone strategies. The structure of equilibrium is slightly different in comparison to the (first price) all-pay auction. In the war of attrition, it is possible to have a nonmonotone equilibrium where the bidders with high valuations earn a strictly positive information rent.

Besides equilibrium characterization, we examine which game induces the highest aggregate effort. In the auction terminology, this is just the revenue comparison across the auction formats. We determine a revenue ranking between the all-pay auction, war of attrition, and the standard auction formats. Their ranking relies on the differences between the auction formats in how much of the bidders' rents they extract, and in how much of the surplus loss results from inefficient allocations.

In models with monotone equilibria, only the rent extraction matters, and then the ranking from Krishna and Morgan (1997) is maintained: war of attrition dominates the all-pay auction which in turn dominates the standard formats. This result holds also for the mineral rights case of the model where there is no allocative inefficiency. The revenue ranking is based on an unambiguous ranking of bidder rents across the formats: standard auctions leave a higher rent to bidders than all-pay auctions which leave a higher rent than the war of attrition.

When considering non-monotone equilibria in the affiliated private values model, the results change. Since non-monotone equilibria inevitably result in an efficiency loss, the relevant comparison is now between the induced inefficiencies and the changes in bidder rents. We show that with two bidders, the ranking between all-pay auctions and standard auctions is as before. However, the ranking between all-pay auctions and the war of attrition is ambiguous. For large numbers of bidders, the rents given up to the high types vanish in all of these auctions, whereas we show that the inefficiencies in the all-pay auction remain. As a result, the previous ranking between all-pay auctions and standard auctions is reversed when the number of bidders grows large.

\section{Related Literature}

The early literature of all-pay auctions and contests has focused on environments where bidders have complete information about each bidder's value of the object and cost of bidding. Examples of such papers include Hillman and Riley (1989), Baye, Kovenock and de Vries (1993) and Che and Gale (1998). Siegel $(2009,2010)$ provides a definitive treatment of this model by allowing heterogeneity on the bidder's characteristics. The recent survey paper by Kaplan and Zamir (2015) gives a comprehensive picture of recent developments in the all-pay auction and contest theory.

Previous work on all-pay auctions with incomplete information about the valuations has mostly concentrated on models with monotone equilibria. The symmetric model with private values possesses a symmetric monotone equilibrium that can be derived using the revenue

\footnotetext{
${ }^{3}$ See Klemperer and Bulow (1999) for the analysis of an N-player war of attrition with an appropriate modification that restores existence of equilibrium. Krishna and Morgan (1997) analyze a "silent" (static) war of attrition model, where the players do not observe each others' actions during the game.
} 
equivalence theorem. ${ }^{4}$ An early contribution to the affiliated values model by Krishna and Morgan (1997) derives sufficient conditions for the existence of a symmetric monotone equilibrium in pure strategies. Unfortunately, the conditions are very strong and furthermore not easily verified in terms of the primitives of the model. More recently Siegel (2014) analyzes an asymmetric two-bidder game with a finite set of possible signals on the value of the prize, and derives conditions for the existence of a monotone equilibrium in mixed strategies. His paper also demonstrates rent-dissipation under perfect correlation, an effect that plays a key role in our analysis also with less than perfect correlation. Lu and Parreiras (2017) analyzes the asymmetric two-bidder game with a continuum signal structure, derives conditions for the existence of a monotone pure-strategy equilibrium, and provides an example of non-monotonic equilibrium.

The most closely related paper to ours is Rentschler and Turocy (2016). Their paper goes beyond monotonic equilibria in a symmetric version of Siegel (2014) and provides an algorithmic characterization for non-monotone equilibria. In contrast to that paper, we provide a full characterization of the symmetric equilibria for a subclass of models featuring the binary structure of signals and allow for any number of bidders. We also analyze the war of attrition with two players and conduct revenue comparisons with other auction procedures.

Our paper is also related to auctions with entry costs. A recent paper by Murto and Välimäki (2017) compares the expected revenue in first- and second-price common value auctions when prior to the auction stage, the bidders make a costly entry decision. The connection to the current non-standard auction forms comes from the observation that the total payment by losing bidders is positive even in these standard auction formats once we account for the entry cost.

\section{The Model}

We consider a symmetric affiliated-values model with binary signals. A single indivisible object is auctioned to one of $N \geq 2$ risk-neutral bidders. Prior to bidding, each bidder $i$ privately observes a binary signal (or type) $t_{i} \in\{L, H\}$. We order the signals $H>L$ with the idea that $H$ (the high type) is good news about the value of the object for sale compared to $L$ (the low type).

The signals are affiliated in the sense of Milgrom and Weber (1982) with a common unknown factor $\theta \in \Theta=\left\{\theta_{1}, \cdots, \theta_{M}\right\}$, which we call the state of the world and arrange in order of $\theta_{m-1}<$ $\theta_{m}$. Specifically, the signals are identically and independently distributed given $\theta$. Due to the binary structure, we can represent the conditional distribution of $t_{i}$ by vector $\left(\alpha_{1}, \cdots, \alpha_{M}\right)$, where $\alpha_{m}:=\operatorname{Pr}\left(t_{i}=H \mid \theta_{m}\right)$. Since $t_{i}$ and $\theta$ are affiliated, $\alpha_{m}$ is increasing in the index of the state $m$. Let $q(\theta) \in \Delta(\Theta)$ denote the common prior on the state. With a slight abuse of notation, we denote by $q(\theta \mid \mathbf{t})$ and $q_{t_{i}}(\theta)$ the posterior belief about the state given a vector of signals $\mathbf{t}$ and an individual signal $t_{i}$, respectively. ${ }^{5}$

\footnotetext{
${ }^{4}$ In the two-bidder asymmetric environment where the revenue equivalence theorem is not applicable, Amann and Leininger (1996) provides a characterization of equilibrium and also studies a hybrid auction which postulates that the lower pays his bid and the winner pays a convex combination of the two bids.

${ }^{5}$ Bold notation will be used throughout the paper to represent vectors of signals, bids, and strategies.
} 
The value of the object to bidder $i$ is determined by the informational variables introduced above and denoted by $v_{i}(\theta, \mathbf{t})$. We assume that the bidders are ex ante symmetric, and that the bidder $i^{\prime}$ s valuation depends only on $\theta$ and $t_{i}$ in the same manner:

$$
v_{i}(\theta, \mathbf{t})=v\left(\theta, t_{i}\right)
$$

The most important special cases of our model are the mineral rights model where $v\left(\theta, t_{i}\right)=v(\theta)$ and the affiliated private values model where $v\left(\theta, t_{i}\right)=v_{t_{i}}$. In addition, we assume that $v\left(\theta, t_{i}\right)$ is log-supermodular in $\left(\theta, t_{i}\right)$, increasing in each argument, and strictly positive for all possible realizations of $\left(\theta, t_{i}\right){ }^{6}$

Since the bidders are ex ante symmetric, all payoff-relevant information to bidder $i$ is abbreviated to $\left(t_{i}, Y_{i}\right)$, where $Y_{i}$ indicates the number of opponents $j \neq i$ who receive the high signal. Conditional on this relevant information, the expected value to bidder $i$ can be then written in a succinct way:

$$
V_{k}(n):=\mathbb{E}_{\theta}\left[v\left(\theta, t_{i}\right) \mid Y_{i}=n, t_{i}=k\right] .
$$

Since $v$ is increasing and the signals are affiliated, $V_{k}(n)$ is increasing in $k$ and $n$. Using the same notation as the expected value, let $p_{k}(n):=\operatorname{Pr}\left(Y_{i}=n \mid t_{i}=k\right)$ denote bidder $i$ 's updated beliefs about $Y_{i}$ after observing $t_{i}$. Affiliation implies that $p_{k}(n)$ is log-supermodular. ${ }^{7}$

Within this informational model, we analyze auctions in which the highest bid wins and a bidder's payment depends only on the profile of bids $\mathbf{b}=\left(b_{1}, \cdots, b_{N}\right)$. Formally, in auction $\Gamma$, bidder i's payment is $\bar{X}_{i}^{\Gamma}(\mathbf{b})$ if he wins and $\underline{X}_{i}^{\Gamma}(\mathbf{b})$ if he loses. As the actual submitted bid depends on the type of a bidder, we represent a strategy of bidder $i$ by a pair of cumulative distribution functions $\mathbf{F}_{i}=\left(F_{i}^{L}, F_{i}^{H}\right)$, where $F_{i}^{k}(b)=\operatorname{Pr}\left(b_{i} \leq b \mid t_{i}=k\right)$.

In an all-pay auction $(\Gamma=A)$, a highest bidder wins the auction and all bidders forfeit their bids. ${ }^{8}$ A war of attrition (W) is a two-player auction where a highest bidder wins and pays the bid of the losing bidder while the losing bidder pays his own bid. We compare these two allpay auction formats to the standard first-price (FP) and second-price auctions (SP) where only the winner is required to pay. In each auction format $\Gamma \in\{\mathrm{A}, \mathrm{W}, \mathrm{FP}, \mathrm{SP}\}$, the (ex post) payoff function

\footnotetext{
${ }^{6}$ The assumption of log-supermodular $v\left(\theta, t_{i}\right)$ is trivially satisfied in the mineral rights model and in the affiliated private values model.

${ }^{7}$ As discussed in the introduction, the state variable $\theta$ has different interpretations depending on the model. In the mineral rights model, $\theta$ represents the true value of the object. In the affiliated private values model, $\theta$ corresponds to a parameter that determines the true distribution of the bidder types. Compared to the reduced-form where bidder $i$ 's interdependent valuation is given by $V_{t_{i}}(n)$, this state formulation plays two roles in our analysis of the all-pay auction in Section 3. First, it allows us to do comparative static analysis on equilibrium with respect to the number of bidders, without any changes in the underlying type distributions. Second, the state formulation equips our environment with the single-crossing property (See Lemma 2) that is essential for a full characterization of equilibrium in Proposition 2. Even in the reduced-form model, the characterization result holds so long as we keep the single-crossing property. See our discussion subsequent to Lemma 2 and also Footnote 12.

${ }^{8}$ If multiple bidders submit the highest bid, we assume that the good is allocated with the standard tie-breaking rule.
} 
to bidder $i$ conditional on all the information $(\theta, \mathbf{t})$ can be written as

$$
\widetilde{u}_{i}^{\Gamma}(\mathbf{b} ; \theta, \mathbf{t})= \begin{cases}v\left(\theta, t_{i}\right)-\bar{X}_{i}^{\Gamma}(\mathbf{b}) & \text { if wins } \\ \underline{X}_{i}^{\Gamma}(\mathbf{b}) & \text { if loses. }\end{cases}
$$

At the moment of bidding, bidder $i$ neither observes the value of $\theta$ nor $\mathbf{t}_{-i}$. Based on the information $t_{i}=k$ available to bidder $i$, his expected payoff from bidding an amount of $b$ when his opponents play according to the strategy $\mathbf{F}_{-i}$ is written as

$$
u_{i}^{\Gamma}\left(b, k \mid \mathbf{F}_{-i}\right)=\mathbb{E}\left[\widetilde{u}_{i}^{\Gamma}(\mathbf{b} ; \theta, \mathbf{t}) \mid t_{i}=k, b_{i}=b, \mathbf{b}_{-i} \sim \mathbf{F}_{-i}\right] .
$$

We analyze Bayes Nash equilibria of the auctions. Formally, a strategy profile $\mathbf{F}=\left(\mathbf{F}_{1}, \cdots, \mathbf{F}_{N}\right)$ constitutes an equilibrium of auction $\Gamma$ if for each bidder $i$ and each type $k$,

$$
b_{i} \in \operatorname{int}\left(\operatorname{supp}\left[F_{i}^{k}\right]\right) \text { implies } b_{i} \in \underset{b}{\operatorname{argmax}} u_{i}^{\Gamma}\left(b, k \mid \mathbf{F}_{-i}\right),
$$

where $\operatorname{supp}\left[F_{i}^{k}\right]$ indicates the support of the bid distribution function $F_{i}^{k}$. The minimum and maximum element of supp $\left[F_{i}^{k}\right]$ will be denoted by $\underline{B}_{k}$ and $\bar{B}_{k}$, respectively. ${ }^{9}$

We say that an equilibrium $\mathbf{F}$ is symmetric if all bidders with identical signals employ the same bidding strategy: $\mathbf{F}_{i}=\mathbf{F}_{*}=\left(F_{*}^{L}, F_{*}^{H}\right)$ for all $i$. Throughout the paper, we restrict attention to symmetric equilibria and use the asterisk subscript for a symmetric equilibrium or equilibrium payoff. Also, we say that a symmetric equilibrium $\mathbf{F}_{*}$ is in monotone strategies if for any $b \in$ $\operatorname{supp}\left[F_{*}^{L}\right]$ and $b^{\prime} \in \operatorname{supp}\left[F_{*}^{H}\right]$, we have $b \leq b^{\prime}$. In a monotone equilibrium, therefore, a high-type bidder wins against low-type ones with probability one.

In a symmetric equilibrium, all bidders of the same type obtain the same expected payoff. We denote by $U_{*}^{\Gamma}(k)$ the symmetric equilibrium payoff of type $k$ in auction $\Gamma$ and refer to it as the information rent. Since all bids in the interior of supp $\left[F_{*}^{k}\right]$ must yield the same payoff to type $k$, we have

$$
U_{*}^{\Gamma}(k):=u_{i}^{\Gamma}\left(b, k \mid \mathbf{F}_{*}\right) \quad \text { for every } b \in \operatorname{int}\left(\operatorname{supp}\left[F_{*}^{k}\right]\right) .
$$

Lastly, we denote by $\Pi_{*}^{\Gamma}$ the expected revenue from auction $\Gamma$ accruing to the auctioneer in a symmetric equilibrium. A standard way to compute the expected revenue is to subtract the bidders' aggregate rents from the total surplus generated by the auction. However, as we will show in what follows, the auctions may entail different equilibrium allocations and therefore different total surpluses. In order to deal with this issue, we first define $\bar{\Pi}$ as the expected total surplus under the efficient allocation:

$$
\bar{\Pi}:=\mathbb{P}(0) V_{L}(0)+\sum_{n=1}^{N} \mathbb{P}(n) V_{H}(n-1),
$$

\footnotetext{
${ }^{9}$ Recall that the support of a probability distribution is defined as the smallest closed set with full measure. Hence the two boundary points, $\underline{B}_{k}$ and $\bar{B}_{k}$, are elements of supp $\left[F_{i}^{k}\right]$.
} 
where $\mathbb{P}(n)$ indicates the probability of $n=0, \cdots, N$ high-type bidders present in the auction. We then subtract away the inefficiency loss and the bidders' rents to compute the revenue. For the former, observe that a misallocation occurs in our model when a low-type bidder outbids hightype ones and leads to a surplus loss of $V_{H}(n-1)-V_{L}(n)$ when $n \geq 1$ high types are present in the auction. Hence we can express the expected surplus loss as

$$
L_{*}^{\Gamma}:=\sum_{n=1}^{N} \mathbb{P}(n) \operatorname{Pr}(\mathrm{L} \text { wins } \mid \Gamma, n)\left(V_{H}(n-1)-V_{L}(n)\right),
$$

where $\operatorname{Pr}(\mathrm{L}$ wins $\mid \Gamma, n)$ represents the probability of the misallocation conditional on the number of high types $n$ in auction $\Gamma$. The bidders' aggregate expected payoff can be expressed as as

$$
U_{*}^{\Gamma}=\sum_{n=0}^{N} \mathbb{P}(n)\left((N-n) U_{*}^{\Gamma}(L)+n U_{*}^{\Gamma}(H)\right) .
$$

With these definitions in hand, we can compute the expected revenue from auction $\Gamma$ as

$$
\Pi_{*}^{\Gamma}=\bar{\Pi}-L_{*}^{\Gamma}-U_{*}^{\Gamma}
$$

Note that the term $\bar{\Pi}$ does not depend on the auction format. Therefore, we can examine the performance of auctions by comparing the efficiency loss $L_{*}^{\Gamma}$ and the rents accruing to bidders $U_{*}^{\Gamma}$ across auction formats.

For the all-pay auction and the war of attrition, it may be more natural to interpret payments as effort expended when competing for a prize. Hence the comparison of expected revenues can be seen as comparison between expected aggregate effort. In a similar vein, the revenues in standard auction formats (first- and second-price auctions) can be interpreted as commitments to effort levels conditional on winning the contest.

\subsection{Benchmark: Standard Auctions}

As a benchmark case, we report here the equilibrium properties of the first- and second-price auctions in our environment. We prove in the Online Appendix that both auction formats have a unique symmetric equilibrium in monotone strategies. Furthermore, the two auction formats are payoff equivalent.

Proposition 1. Both standard auctions have a unique symmetric equilibrium, which is in monotone strategies. Specifically,

(FP) in the equilibrium of the first-price auction, the low type bids $V_{L}(0)$ and the high type draws a bid according to an atomless distribution on $\left[V_{L}(0), \mathbb{E}\left[v\left(\theta, t_{i}\right) \mid t_{i}=H\right]-p_{H}(0)\left(V_{H}(0)-V_{L}(0)\right)\right]$;

(SP) in the equilibrium of the second-price auction, the low type bids $V_{L}(0)$ and the high type draws a bid according to an atomless distribution on $\left[V_{H}(0), \mathbb{E}\left[v\left(\theta, t_{i}\right) \mid t_{i}=H, Y_{i} \geq 1\right]\right]$. 
In both auction formats, the low-type bidders earn no rent but the high-type bidders earn a positive rent of $p_{H}(0)\left(V_{H}(0)-V_{L}(0)\right)$.

Proof. The proof of Proposition 1 can be found in the Online Appendix.

Since all bidders receive the same equilibrium payoff in both auctions, we shall use the same superscript $S$ for standard auctions when describing bidders' rent and seller's revenue. Intuitively, bidder $i$ gets a positive rent only if he is the only one with high type (i.e., with probability $p_{H}(0)$ ), and then his rent equals his value minus the bid of low types. Utilizing the payoff equivalence result, we can compute the aggregate information rents appropriated by the bidders in the standard auctions as follows:

$$
\begin{aligned}
U_{*}^{S}=\sum_{n=0}^{N} n \mathbb{P}(n) U_{*}^{S}(H) & =N p_{H}(0) \sum_{n=1}^{N} \operatorname{Pr}\left(t_{i}=H, Y_{i}=n-1\right)\left(V_{H}(0)-V_{L}(0)\right) \\
& =\mathbb{P}(1)\left(V_{H}(0)-V_{L}(0)\right) .
\end{aligned}
$$

The last two expressions are derived from the fact that the joint probability $\operatorname{Pr}\left(t_{i}=H, Y_{i}=n-1\right)$ and the ex-ante probability $\mathbb{P}(n)$ are interrelated by

$$
\frac{N}{n} \cdot \operatorname{Pr}\left(t_{i}=H, Y_{i}=n-1\right)=\mathbb{P}(n) \quad \forall n=1, \cdots, N
$$

Another implication of Proposition 1 is that as both standard auctions have a unique symmetric equilibrium in monotone strategies, there is no surplus loss from misallocation, i.e., $L_{*}^{S}=0$. Hence we can compute the expected revenue from the standard auctions as follows:

Corollary 1. Both standard auctions are equivalent in terms of the expected revenue accruing to the auctioneer:

$$
\Pi_{*}^{S}=\bar{\Pi}-\mathbb{P}(1)\left(V_{H}(0)-V_{L}(0)\right) .
$$

It is also worth nothing that this revenue equivalence result is specific to the binary signal structure. ${ }^{10}$ When we allow a richer signal space, it follows from the linkage principle (Milgrom and Weber (1982)) that the second-price auction results in a higher expected revenue than the first-price auction.

\section{All-pay Auction}

In a first-price all pay auction, all bids are forfeited by the bidders regardless of the outcome: $\underline{X}_{i}^{A}(\mathbf{b})=\bar{X}_{i}^{A}(\mathbf{b})=b_{i}$ for all $i$.

\footnotetext{
${ }^{10}$ Within the two-bidder binary private-value model, Maskin and Riley (1985) have shown that revenue equivalence holds between the first- and second-price (equivalently, the open ascending price) auctions.
} 


\subsection{Equilibrium Characterization}

We begin our analysis with two observations providing necessary conditions for any symmetric equilibria $\mathbf{F}_{*}$ in the all-pay auction. First, there cannot be atoms in the bid distribution. If $F_{*}^{k}$ has an atom at bid $b$, i.e., if the probability of winning jumps up at $b$, then a type- $k$ bidder could raise the expected payoff by bidding slightly more than $b$. Second, the union of the two bid supports, $\operatorname{supp}\left[F_{*}^{L}\right] \cup \operatorname{supp}\left[F_{*}^{H}\right]$, should be an interval starting at zero with no internal gaps. If no bidders make a bid between $b_{1}$ and $b_{2}>b_{1}$, the bidder who submitted $b_{2}$ could reduce his payment by deviating to $b_{1}$ without any changes in the probability of winning.

Lemma 1. In every symmetric equilibrium $\mathbf{F}_{*}=\left(F_{*}^{L}, F_{*}^{H}\right)$ of the all-pay auction, the following properties hold:

1. For each type $k=L, H, F_{*}^{k}$ is continuous, i.e., neither distribution has mass points.

2. $\operatorname{supp}\left[F_{*}^{L}\right] \cup \operatorname{supp}\left[F_{*}^{H}\right]$ is a connected interval that includes zero.

Suppose that bidder $i$ observes $t_{i}=k$ and makes a bid of $b \geq 0$ against his opponents who each employ a symmetric equilibrium strategy $\mathbf{F}_{*}$. An implication of Lemma 1 is that bidder $i^{\prime} s$ expected payoff is continuous at his bid $b$ and thus is written as

$$
u^{A}\left(b, k \mid \mathbf{F}_{*}\right):=-b+\sum_{n=0}^{N-1} V_{k}(n) p_{k}(n)\left[F_{*}^{H}(b)\right]^{n}\left[F_{*}^{L}(b)\right]^{N-n-1}
$$

To interpret, $V_{k}(n)$ represents the expected value of the object to the bidder conditional on winning against $Y_{i}=n$ high-type opponents, and $\left[F_{*}^{H}(b)\right]^{n}\left[F_{*}^{L}(b)\right]^{N-n-1}$ indicates the corresponding probability of winning the auction.

The bulk of the literature on all-pay auctions has concentrated on monotone equilibria in which the high-type bidders always outbid the low-type ones. We provide a simple condition under which this is the case in our binary model. We also characterize the non-monotone equilibrium that arises when this monotonicity condition is violated. The simple information structure enables us to give a full characterization of a symmetric equilibrium in either case and to establish its existence and uniqueness.

For this purpose, we define a real-valued function $\psi$ by $\psi(n):=V_{H}(n) p_{H}(n)-V_{L}(n) p_{L}(n)$. Given the number of high-type opponents being $n$, the defined function measures the increment in the value of $V_{k}(n) p_{k}(n)$ in response to a change of bidder $i$ ' type from $k=L$ to $H$. We demonstrate that under the assumptions we made in the previous section, $\psi(n)$ changes its sign at most once from negative to positive. This single-crossing property plays a central role in our equilibrium analysis. In particular, the property ensures that each of the bidding supports in $\mathbf{F}_{*}$ is a connected interval, as is shown in the proof of Proposition 2.

To see how the sign of $\psi$ changes over $n$, observe that $V_{H}(n) \geq V_{L}(n)$ for every $n$ and $p_{H}(N-$ 1) $>p_{L}(N-1)$ due to affiliation, implying that $\psi(N-1)>0$ at least. In case of two bidders, 
therefore, the single-crossing property holds trivially. ${ }^{11}$ For the general case, affiliation implies that $p_{H}(n) / p_{L}(n)$ is increasing in $n$, and hence $\psi$ is single-crossing unless the ratio $V_{H}(n) / V_{L}(n)$ decreases sharply over some range of $n$. In fact, our assumption of log-supermodular $v\left(\theta, t_{i}\right)$ precludes the possibility of decreasing $V_{H}(n) / V_{L}(n)$. Since log-supermodularity is preserved by integration (Karlin and Rinott (1980)), the assumption leads to log-supermodular $V_{k}(n)$ and thus the desired property of $\psi$ follows. ${ }^{12}$

Lemma 2. If the bidder's valuation $v\left(\theta, t_{i}\right)$ is log-supermodular, then $\psi(n)$ is single-crossing.

Proof. See Appendix A.1.

The next proposition establishes the uniqueness of a symmetric equilibrium $\mathbf{F}_{*}$, provides a necessary and sufficient condition for $\mathbf{F}_{*}$ to be in monotone strategies, and characterizes both monotone and non-monotone equilibria.

Proposition 2. The all-pay auction has a unique symmetric equilibrium $\mathbf{F}_{*}$, which is monotonic if and only if $\psi(0) \geq 0$. Specifically,

(i) If $\psi(0) \geq 0$, then $\mathbf{F}_{*}$ has the two bidding supports connected and non-overlapping:

$$
\operatorname{supp}\left[F_{*}^{L}\right]=\left[0, V_{L}(0) p_{L}(0)\right] \text { and } \operatorname{supp}\left[F_{*}^{H}\right]=\left[V_{L}(0) p_{L}(0), \bar{B}_{H}\right] \text { for some } \bar{B}_{H}>V_{L}(0) p_{L}(0) \text {. }
$$

(ii) If $\psi(0)<0$, then $\mathbf{F}_{*}$ has the two bidding supports connected and fully overlapping:

$$
\operatorname{supp}\left[F_{*}^{L}\right]=\left[0, \bar{B}_{L}\right] \text { and } \operatorname{supp}\left[F_{*}^{H}\right]=\left[0, \bar{B}_{H}\right] \text { for some } 0<\bar{B}_{L}<\bar{B}_{H} \text {. }
$$

Consequently, the low-type bidders earn no rent $U_{*}^{A}(L)=0$ in both types of equilibria, whereas the hightype bidders earn a rent of $U_{*}^{A}(H)=\max \{0, \psi(0)\}$.

Proof. See Appendix A.2.

Proposition 2 tells us that it hinges upon the sign of $\psi(0)$ whether the unique equilibrium is monotone or not. By Lemma 2, $\psi(0) \geq 0$ implies $\psi(n) \geq 0$ for all $n$ which in turn ensures that for a fixed bid, the high type obtains a higher expected gain of winning than the low type, regardless of the number of high-type opponents. For this reason, the event $t_{i}=H$ becomes unambiguously good news relative to $t_{i}=L$ in our model. To understand better why this results in a monotone equilibrium, consider a monotone bidding strategy $\mathbf{F}_{*}$ where the low type randomizes over $\left[0, \bar{B}_{L}\right]$ and the high type randomizes over $\left[\underline{B}_{H}, \bar{B}_{H}\right]$ with $\underline{B}_{H} \geq \bar{B}_{L}$. For $\mathbf{F}_{*}$ to be an equilibrium, the union

\footnotetext{
${ }^{11}$ Accordingly, in the two-bidder case, we can abstract away several assumptions on the primitive model, such as the conditional independence of types on $\theta$ and the log-supermodularity of $v\left(\theta, t_{i}\right)$. For more than two bidders, our analysis and subsequent results can be extended to the general symmetric interdependent valuations, as long as $\psi$ is single-crossing.

${ }^{12}$ To see one example where $\psi$ does not satisfy the single-crossing property, consider $V_{k}(n)=\alpha \mathbb{1}_{\{k=H\}}+(1-\alpha) n+\epsilon$, where $\epsilon>0$ is a sufficiently small constant and $\alpha \geq \frac{1}{2}$ and $\mathbb{1}_{\{k=H\}}$ is the indicator function of the event that $t_{i}=k$. Then the ratio $V_{H}(n) / V_{L}(n)$ drops drastically, especially when $n$ increases from 0 to 1 . As a result, if $p_{H}(0) / p_{L}(0)>\epsilon /(\alpha+\epsilon)$ but $p_{H}(1) / p_{L}(1)<(1-\alpha+\epsilon) /(1+\epsilon)$, then we have $\psi(0)>0$ but $\psi(1)<0$.
} 


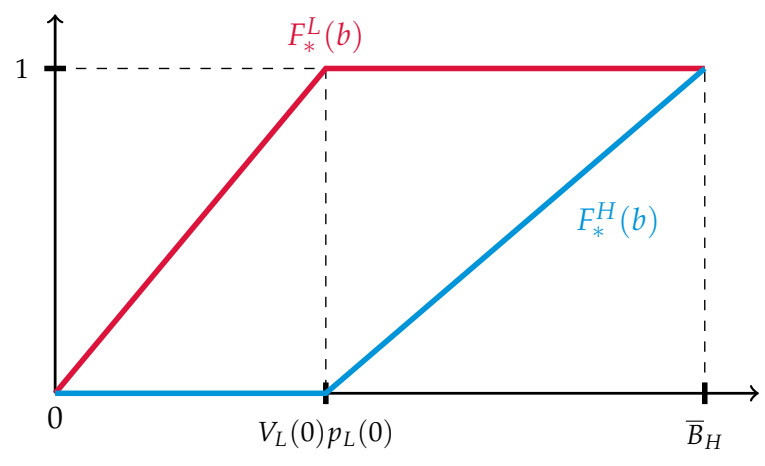

(a) monotonic equilibrium when $\psi(0) \geq 0$

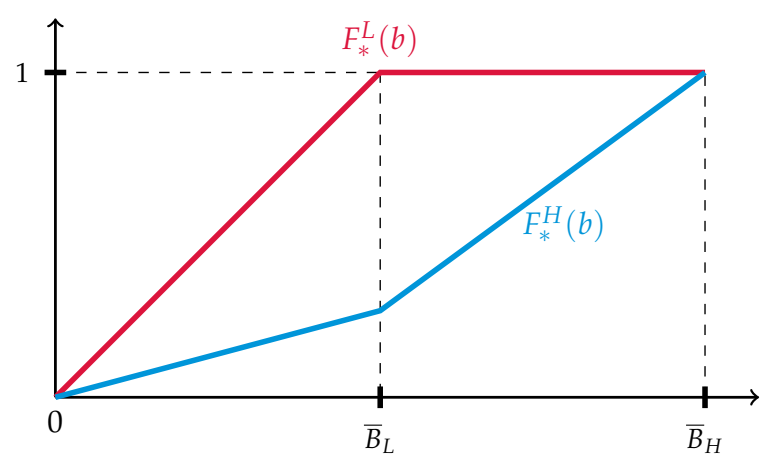

(b) non-monotonic equilibrium when $\psi(0)<0$

Figure 1: Symmetric equilibrium in case of two bidders

of the two bidding supports should form a connected interval by Lemma 1 , and thus $\underline{B}_{H}=\bar{B}_{L}$. Furthermore, the high type must make a non-negative payoff by bidding $\bar{B}_{L}$, for otherwise she would deviate to making a bid of zero. By bidding zero, the low type never wins and hence his expected payoff should be zero. On the other hand, by bidding $\bar{B}_{L}$, the low type wins with probability $p_{L}(0)$ (that is, when all of his opponents are of low type) and receives a payoff of $V_{L}(0)$ so that his expected gain is $V_{L}(0) p_{L}(0)$. Equating this gain with his bid so as to guarantee the same expected payoff from bidding zero, we have

$$
\bar{B}_{L}=V_{L}(0) p_{L}(0)
$$

Consider next the high-type bidder's incentives. By making bid $\bar{B}_{L}$, the high type wins with probability $p_{H}(0)$ and receives a payoff of $V_{H}(0)$. Therefore, $\bar{B}_{L}$ yields a non-negative payoff to the high type iff $V_{H}(0) p_{H}(0) \geq \bar{B}_{L}$, or iff $\psi(0) \geq 0$. This demonstrates that $\psi(0) \geq 0$ is a necessary condition for the monotone strategy $\mathbf{F}_{*}$ to constitute an equilibrium. Conversely, the condition is also sufficient for $\mathbf{F}_{*}$ to be monotonic. Specifically, it can be shown that if $\psi(0) \geq 0$, then the bidder's expected payoff function $u^{A}\left(b, k \mid \mathbf{F}_{*}\right)$ in (1) exhibits strictly increasing differences in $(b ; k)$ for all $\mathbf{F}_{*}$ satisfying the two properties of Lemma 1 . This notion of complementarity results in the unique symmetric equilibrium being monotonic.

Figure 1 displays the different types of equilibria in two-bidder case. The left panel shows the monotonic equilibrium that arises when $\psi(0) \geq 0$, which is qualitatively similar to the equilibrium in an all-pay auction with independent private values. In this equilibrium, each bidder competes against bidders of her own type and a bid of zero is in the support of the low type only. As a result, the low type makes no positive rents whereas the high type earns a positive rent. Since the net profit of increasing a bid by $\Delta$ must be constant within each support, and since the corresponding benefit and cost are additively separate in the all-pay auction, the equilibrium bid distributions must be uniform on the support of each type.

The right panel of Figure 1 displays a non-monotonic equilibrium. It is worthwhile to note that in this type of equilibrium, the two bidding supports are fully overlapping and both include 
the bid of zero. As an immediate consequence, the equilibrium involves full rent dissipation. This is in sharp contrast with the standard result in allocation problems under asymmetric information that due to informational advantages (and privacy), the arrival of good news leaves a positive rent to agents. To grasp the underlying intuition, observe that the payoff function is linear in the choice variable $b$. In order to satisfy the indifference condition within a support, the winning probability must change linearly in bids due to the uniform all-pay rule. Hence the bidder's best response has the bang-bang property: if the bid $\bar{B}_{L}$ on the boundary between the bid supports does not yield a non-negative payoff to a high-type bidder, then she would respond by bidding zero.

We conclude this section by analyzing which type of equilibrium we should expect as the number of participants $N$ increases. To this end, we rewrite the monotonicity condition $\psi(0) \geq 0$ as

$$
\frac{V_{H}(0)}{V_{L}(0)} \geq \frac{p_{L}(0)}{p_{H}(0)}
$$

It is immediate from (2) that in case of independent types, the ratio $p_{L}(0) / p_{H}(0)$ is equal to one regardless of $N$, so the unique symmetric equilibrium is monotone even when a large number of bidders compete in the auction. However, since $p_{L}(0)$ approaches zero in the limit as $N \rightarrow \infty$, the length of supp $\left[F_{*}^{L}\right]=\left[0, V_{L}(0) p_{L}(0)\right]$ shrinks to zero and thus only the high types submit nonzero bids.

To examine how correlated types affect the form of equilibrium, consider first the mineral rights model where the bidder's common valuation depends only on $\theta$. In this model, the impact of an individual's signal on the value of the object naturally diminishes as $N$ grows, and hence the ratio $V_{H}(0) / V_{L}(0)$ on the left-hand side of (2) converges to one. On the other hand, the ratio $p_{L}(0) / p_{H}(0)$ on the other side converges to $q_{L}\left(\theta_{1}\right) / q_{H}\left(\theta_{1}\right)$, where $q_{k}\left(\theta_{1}\right) \equiv \operatorname{Pr}\left(\theta=\theta_{1} \mid t_{i}=k\right)$, i.e. the likelihood ratio of the lowest possible state across the two types. Intuitively, only the lowest state $\theta_{1}$ matters for the ratio $p_{L}(0) / p_{H}(0)$ as $N \rightarrow \infty$, in the sense that the likelihood of $Y_{i}=0$ reduces to zero at a higher rate in all other states. Let $p_{H}=\sum_{m=1}^{M} q\left(\theta_{m}\right) \alpha_{m}$ denote the unconditional prior probability of getting a high signal. By Bayes' rule,

$$
\frac{q_{L}\left(\theta_{1}\right)}{q_{H}\left(\theta_{1}\right)}=\frac{1-\alpha_{1}}{\alpha_{1}} \cdot \frac{p_{H}}{1-p_{H}}>1,
$$

where the last inequality follows from $p_{H}>\alpha_{1}$. As a result, the inequality in (2) is violated as $N \rightarrow \infty$, showing that the equilibrium tends to be non-monotone for large $N$.

With affiliated private values, the left-hand side of (2) is constant at $v_{H} / v_{L}>1$. In the proof of Proposition 3, we show that the right-hand side is increasing in $N$ with limit $q_{L}\left(\theta_{1}\right) / q_{H}\left(\theta_{1}\right)>1$. Consequently, which type of equilibrium arises for large $N$ hinges upon the comparison of $v_{H} / v_{L}$ and $q_{L}\left(\theta_{1}\right) / q_{H}\left(\theta_{1}\right)$. The following proposition summarizes our discussion.

Proposition 3. Fix the information structure $\left\langle q,\left(\alpha_{1}, \cdots, \alpha_{M}\right)\right\rangle$ and the bidder's valuation $v\left(\theta, t_{i}\right)$. The unique symmetric equilibrium $\mathbf{F}_{*}$ satisfies:

† In the mineral rights model, there exists a $\bar{N}<\infty$ such that $\mathbf{F}_{*}$ is non-monotonic for all $N>\bar{N}$. 
$\ddagger$ In the affiliated private value model, if $v_{H} q_{H}\left(\theta_{1}\right)>v_{L} q_{L}\left(\theta_{1}\right)$, then $\mathbf{F}_{*}$ is monotonic for all $N$. Otherwise, there exists a $\widehat{N}$ such that $\mathbf{F}_{*}$ is non-monotonic for all $N>\widehat{N}$.

Proof. The proof of Proposition 3 can be found in the Online Appendix.

\subsection{Revenue Properties}

We now turn to the revenue and efficiency properties of the equilibrium. We want to contrast the allocation and expected total revenue in the unique equilibrium of the all-pay auction to those of the standard auction formats.

Propositions 1 and 2 characterize the unique symmetric equilibrium in standard auctions and all-pay auctions, respectively. In both auction procedures, the low-type bidders get no rent as expected, whereas the high-type bidders obtain a positive rent. For a comparison, observe that

$$
p_{H}(0)\left(V_{H}(0)-V_{L}(0)\right)>p_{H}(0) V_{H}(0)-p_{L}(0) V_{L}(0)=\psi(0),
$$

where the inequality follows from $p_{L}(0)>p_{H}(0)$. This means that compared to the all-pay auction, the standard auctions give away a higher rent to the high type: $U_{*}^{S}(H)>U_{*}^{A}(H)$. It leads us to the following lemma:

Lemma 3. The expected rent accruing to bidders is strictly higher in the standard auction formats than in the all-pay auction, $U_{*}^{S}>U_{*}^{A}$.

We next turn to the comparison of allocations across the auction formats. Since the equilibrium in the standard auctions is in monotone strategies, it induces the efficient allocation. In contrast, in the all-pay auction the equilibrium may be non-monotonic in the sense that the bidding supports of the two types overlap. In such a case, there is a positive probability that a low type wins even when high-type bidders are present, thereby leading to an inefficient allocation.

Nevertheless, there are two situations where the all-pay auction achieves allocative efficiency. First, when $\psi(0) \geq 0$, the equilibrium is monotone by Proposition 2. As seen in (2), this is the case when $\frac{V_{H}(0)}{V_{L}(0)}$ is large in comparison to $\frac{p_{L}(0)}{p_{H}(0)}$, in other words, when the effect of own signal on the estimated value is large in comparison to the affiliation effect. Second, if the informational type of the winner does not matter for efficiency, then even a non-monotone equilibrium leads to efficient allocation. This is the case in the mineral-rights model.

Whenever auctions yield an efficient allocation in equilibrium, the task of comparing revenues from these auctions is equivalent to that of comparing the rents accruing to each type of bidders. The next result is thus immediate from Lemma 3:

Proposition 4. If the equilibrium allocation is efficient in the all-pay auction, then the revenue to the seller is higher in the all-pay auction than in the standard auctions. This is the case if either:

- $v\left(\theta, t_{i}\right)=v(\theta)$ (Mineral rights model) or

- $\psi(0) \geq 0$ (Monotonic equilibrium). 
Note that the second case in the proposition corresponds to the result obtained in Krishna and Morgan (1997), which analyzes the symmetric interdependent values model under a continuum signal space but under a parameter restriction that rules out non-monotonic cases. ${ }^{13}$

The revenue comparison becomes more tricky when the all-pay auction features allocative inefficiency. This is the case when $v\left(\theta, t_{i}\right)$ also depends on $t_{i}$ and $\psi(0)<0$. We know from Proposition 2 that whenever $\psi(0)<0$, the bidders' rents are fully dissipated. In this case, therefore, the revenue comparison boils down to comparing the revenue loss due to inefficient allocation in the all-pay auction and the revenue loss due to bidders' rents in the standard auctions.

To illustrate this comparison, consider first the two-bidder case in which the inefficient allocation occurs only when one low and high type compete against each other. Denote by $\mathbb{P}(1)$ the probability of the event that only one high type is present in the auction. The misallocation indeed occurs when the low type outbids the high type, which takes place with probability $\operatorname{Pr}\left(b_{L}>b_{H}\right)$ in the non-monotonic equilibrium, resulting in a reduction of the total surplus by $V_{H}(0)-V_{L}(1)$. The expected revenue loss due to the inefficiency in the all-pay auction can therefore be written as

$$
\mathbb{P}(1)\left(V_{H}(0)-V_{L}(1)\right) \operatorname{Pr}\left(b_{L}>b_{H}\right)
$$

On the other hand, the revenue loss in the standard auctions arising from information rents given up to the high type amounts to

$$
\mathbb{P}(1)\left(V_{H}(0)-V_{L}(0)\right)
$$

This is clearly strictly larger than the loss in the all-pay auction because $V_{L}(1) \geq V_{L}(0)$ and $\operatorname{Pr}\left(b_{L}>b_{H}\right)<1$. Accordingly, in the two-bidder case, the all-pay auction outperforms the standard auctions even when monotonicity fails. We summarize the above discussion in the following proposition.

Proposition 5. With two bidders, the all pay auction generates a higher expected revenue than the standard auction formats.

When there are more than two bidders and valuations have a private component, the comparison is less straightforward. We compare the expected revenues next in the case where the number of bidders grows large. As is intuitively clear, the bidders' information rents vanish due to increased competition as $N \rightarrow \infty$, irrespective of the auction format. However, as we showed in Proposition 3, whenever $v_{H} q_{H}\left(\theta_{1}\right)<v_{L} q_{L}\left(\theta_{1}\right)$, the equilibrium of the all-pay auction remains non-monotonic even for large $N$. It turns out that whenever this is the case, there is a nonnegligible probability that a low type wins the auction even for arbitrarily large $N$. This implies an efficiency loss and reverses the revenue ranking result of Proposition 5 for large $N$.

Proposition 6. In the case of affiliated private values (i.e. $\left.v\left(\theta, t_{i}\right)=v_{t_{i}}\right)$, two cases arise:

\footnotetext{
${ }^{13}$ More precisely, Krishna and Morgan (1997) compares the first-price auction with the all-pay auction in terms of the expected revenue in the (unique) monotone symmetric equilibrium, and shows that the all-pay auction outperforms. The revenue ranking between the all-pay auction and the second-price auction is ambiguous.
} 
- If $v_{H} q_{H}\left(\theta_{1}\right) \geq v_{L} q_{L}\left(\theta_{1}\right)$, then the revenue is higher in the all-pay auction than in the standard auctions for all $N \geq 2$.

- If $v_{H} q_{H}\left(\theta_{1}\right)<v_{L} q_{L}\left(\theta_{1}\right)$, then the revenue is higher in the standard auctions than in the all-pay auction for $N$ sufficiently large.

Proof. The proof of Proposition 6 can be found in the Online Appendix.

\section{War of Attrition with Two Players}

In this section we study a two-player dynamic contest, the so-called war of attrition where two bidders decide at each point of the game whether to stay in the game for an incremental unit of length $d t$ at a cost of $d t$ or to drop out. The game ends when one of the two players drops out, and the other player wins the contest. As is well-known, this contest can be modeled as a second-price all pay auction with payment rules, $\bar{X}_{i}(\mathbf{b})=b_{j}$ and $\underline{X}_{i}(\mathbf{b})=b_{i}$. With more than two players, the standard (dynamic) war of attrition where the players observe each others' actions during the game has no symmetric equilibrium. Thus, we restrict our attention in this section to a two-player game. The informational structure is exactly the same as before.

\subsection{Equilibrium Characterization}

To begin, we make a small change in the notation suited for a two-player game. For a realization of binary types $\left(t_{i}, t_{j}\right)=(k, m)$, denote by $V_{k}(m)$ the expected value of the object to bidder $i$ and by $p_{k}(m) \equiv \operatorname{Pr}\left(t_{j}=m \mid t_{i}=k\right)$ the bidder $i$ 's posterior belief about $t_{j}$ given his signal. Also, define the product of $V_{k}(m)$ and $p_{k}(m)$ by $\varphi_{k}(m):=V_{k}(m) p_{k}(m)$. That is, we use bidder $i$ 's opponent's type as the main argument instead of the number of high-type opponents for analysis of the war of attrition. To avoid confusion, observe that $t_{j}=L$ corresponds to $Y_{i}=0$ and $t_{j}=H$ corresponds to $Y_{i}=1$.

Let $\mathbf{G}_{*}=\left(G_{*}^{L}, G_{*}^{H}\right)$ be a symmetric strategy profile in the war of attrition, where for each type $k, G_{*}^{k}(b)=\operatorname{Pr}\left(b_{i} \leq b \mid t_{i}=k\right)$ represents the cumulative distribution function for bids. Analogous to the all-pay auction, any symmetric equilibrium $\mathbf{G}_{*}$ must satisfy the two properties stated in Lemma 1. As a result, we can disregard the event of a tie and write bidder 1's expected payoff function as follows:

$$
u^{W}\left(b, k \mid \mathbf{G}_{*}\right)=\mathbb{E}\left[\left(V_{t_{1}}\left(t_{2}\right)-b_{2}\right) \mathbb{1}_{\left\{b_{2} \leq b\right\}}-b \mathbb{1}_{\left\{b_{2}>b\right\}} \mid t_{1}=k, b_{2} \sim \mathbf{G}_{*}\right] .
$$

The following argument presents equilibrium conditions characterizing $\mathbf{G}_{*}$. Consider a bid increment for bidder $i$ with $t_{i}=k$ from $b$ to $b+\Delta$ within the interior of $\operatorname{supp}\left[G_{*}^{k}\right]$. This increment affects bidder $i$ 's payoff only when $b_{j} \geq b$, and bidder $i$ must be indifferent between those two bids. Conditional on $t_{i}=k$ and $b_{j} \sim \mathbf{G}_{*}$, the probability of the event that bidder $j$ with $t_{j}=m$ 
places a bid $b_{j} \in[b, b+\Delta]$ is

$$
\frac{p_{k}(m) g_{*}^{m}(b) \Delta}{1-p_{k}(L) G_{*}^{L}(b)-p_{k}(H) G_{*}^{H}(b)},
$$

and the value from winning the contest to bidder $i$ is $V_{k}(m)$. Hence, if $b$ is in the support of type $k$ but not in the support of type $m \neq k$ (so that $g_{*}^{m}(b)=0$ ), the following condition that equates the marginal cost and benefit from the bid increment must hold: ${ }^{14}$

$$
\frac{\varphi_{k}(k) g_{*}^{k}(b)}{1-p_{k}(L) G_{*}^{L}(b)-p_{k}(H) G_{*}^{H}(b)}=1 .
$$

Therefore, when $b$ is not in the support of type $m \neq k$, the equilibrium condition (DE- 1 ) is given by a linear first-order differential equation for $G_{*}^{k}(b)$.

On the other hand, when the bid $b$ is in the support of both types, the corresponding indifference conditions must hold respectively for the two types:

$$
\begin{gathered}
\frac{\varphi_{L}(L) g_{*}^{L}(b)+\varphi_{L}(H) g_{*}^{H}(b)}{1-p_{L}(L) G_{*}^{L}(b)-p_{L}(H) G_{*}^{H}(b)}=1, \\
\frac{\varphi_{H}(L) g_{*}^{L}(b)+\varphi_{H}(H) g_{*}^{H}(b)}{1-p_{H}(L) G_{*}^{L}(b)-p_{H}(H) G_{*}^{H}(b)}=1 .
\end{gathered}
$$

Consequently, when the two bid supports are overlapped, the equilibrium is characterized by a system of linear differential equations.

With these conditions in hand, we can construct the symmetric equilibrium of the game in the following manner. We start from $b=0$ with the initial condition $G_{*}^{L}(0)=G_{*}^{H}(0)=0$, and then derive the bid distributions from either (DE-1) or (DE-2), depending on whether or not (DE-2) yields a valid solution satisfying $\left(g_{*}^{L}(b), g_{*}^{H}(b)\right) \gg 0$. Figure 2 illustrates the equilibrium construction in the phase plane diagram. It turns out that depending on the primitives of the model, the unique symmetric equilibrium takes one of three different forms as is displayed in Figure 2. This corresponds to three possible equilibrium configurations for the bid supports summarized in Proposition 7 below. The details of the construction and the closed-form solutions for the bid distributions are given in the proof, which is relegated to Appendix A.3. To characterize the equilibrium, we define $\lambda:=p_{H}(H) / p_{L}(H)>1$.

Proposition 7. The war of attrition has a unique symmetric equilibrium $\mathbf{G}_{*}$ with the following properties.

(i) If $\varphi_{H}(L) \geq \lambda \varphi_{L}(L)$, then the equilibrium is in monotone strategies: $\operatorname{supp}\left[G_{*}^{L}\right]=\left[0, \bar{B}_{L}\right]$ and $\operatorname{supp}\left[G_{*}^{H}\right]=\left[\underline{B}_{H}, \infty\right)$ with $\bar{B}_{L}=\underline{B}_{H}$.

(ii) If $\varphi_{H}(L) \in\left[\varphi_{L}(L), \lambda \varphi_{L}(L)\right)$, then $\operatorname{supp}\left[G_{*}^{L}\right]=\left[0, \bar{B}_{L}\right]$ and $\operatorname{supp}\left[G_{*}^{H}\right]=\left[\underline{B}_{H}, \infty\right)$ with $\bar{B}_{L}>$ $\underline{B}_{H}>0$. That is, the two supports are partially overlapping.

\footnotetext{
${ }^{14}$ Notice that whenever $b_{j} \in[b, b+\Delta]$, the true cost $b_{j}$ is within $\Delta$ from $b+\Delta$ and hence the added cost is approximated by $\Delta$.
} 


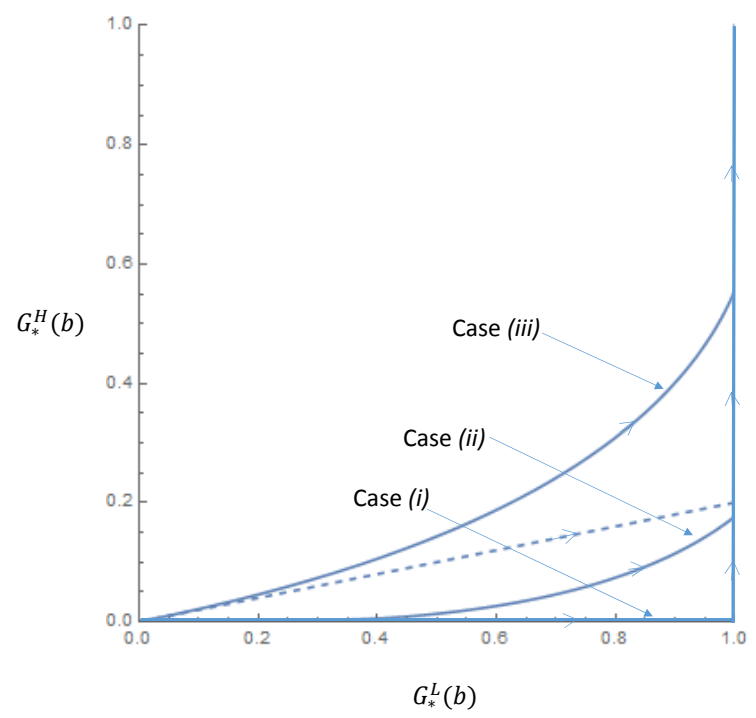

Figure 2: Phase plane diagram of the symmetric equilibrium in the three different cases.

(iii) If $\varphi_{H}(L)<\varphi_{L}(L)$, then $\operatorname{supp}\left[G_{*}^{L}\right]=\left[0, \bar{B}_{L}\right]$ and $\operatorname{supp}\left[G_{*}^{H}\right]=[0, \infty)$.

Proof. See Appendix A.3.

Figure 3 displays the equilibrium bid distributions in the three different cases. These figures are drawn in the case of affiliated private values with different degrees of correlation between the signals. For fixed valuations, the form of the unique equilibrium changes from the case $(i)$ to $(i i)$ and (iii) as the signals become more strongly affiliated.

It is instructive to compare the symmetric equilibrium of the war of attrition and the all-pay auction. In the all-pay auction with two bidders, the monotonicity condition $\psi(0) \geq 0$ can be expressed as $\varphi_{H}(L) \geq \varphi_{L}(L)$ in our current notation. Hence the monotonicity condition identified in Proposition 7 guarantees the existence of a monotone equilibrium in the all-pay auction, which is consistent with Krishna and Morgan (1997).

In addition to the monotonicity condition, the two auction formats exhibit qualitative differences. In the all-pay auction, there are only two different support configurations: either the equilibrium is monotonic (the case corresponding to (i) in Proposition 7) or the supports are fully nested (the case corresponding to (iii)). In Figure 2, the dotted line corresponds to the nonmonotone equilibrium of the all-pay auction. The case (ii), where the two supports are partially overlapping, is hence a distinguishing feature of the war of attrition.

In contrast to the all-pay auction, the indifference condition of the war of attrition results in a constant hazard rate for bids rather than a constant density within each type's bid support, and this gives rise to a more restrictive monotonicity condition. For an illustration, consider a monotone bidding strategy. Substituting $k=L$ and $b=\bar{B}_{L}$ (i.e., $G_{*}^{H}\left(\bar{B}_{L}\right)=0$ ) into the equilibrium 


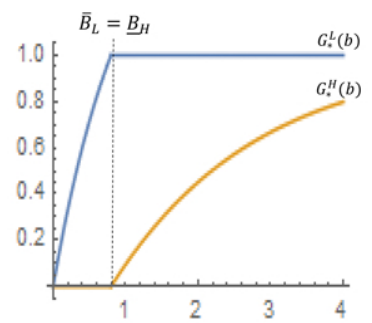

(i)

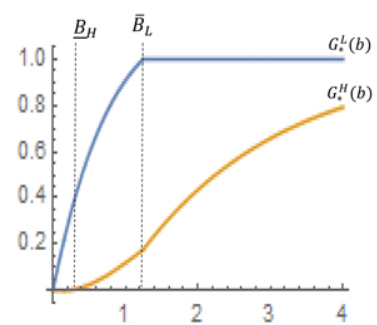

(ii)

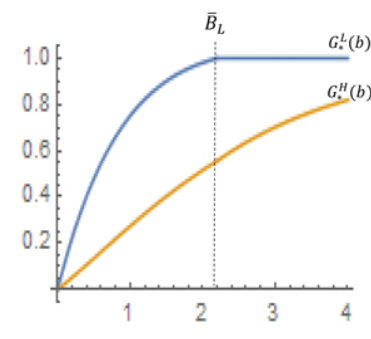

(iii)

Figure 3: Equilibrium bid distributions with private values: $V_{H}=2$ and $V_{L}=1$. The correlation in signals is given by $p_{H}(H)=p_{L}(L)=\gamma$ and $p_{H}(L)=p_{L}(H)=1-\gamma$, where $\gamma=0.55,0.65$, and 0.75 in the left, center, and right panels, respectively.

condition (DE-1) yields

$$
g_{*}^{L}\left(\bar{B}_{L}\right)=\frac{1-p_{L}(L)}{\varphi_{L}(L)} .
$$

To rule out local deviations below $\bar{B}_{L}$ by the high type, we must have:

$$
g_{*}^{L}\left(\bar{B}_{L}\right) \geq \frac{1-p_{H}(L)}{\varphi_{H}(L)}
$$

which, along with (4), gives the condition of case (i) in Proposition 7: $\varphi_{H}(L) \geq \lambda \varphi_{L}(L)$.

\subsection{Revenue Properties}

We now compare the expected revenue from the war of attrition to that from the all-pay auction and standard auctions. As in the all-pay auction, the symmetric equilibrium of the war of attrition is not always in monotone strategies. Hence for a revenue comparison, we analyze how the bidders' rents and allocative inefficiencies differ across the auction formats.

We first consider the bidders' expected rent in the war of attrition. Since a bid of zero belongs to $\operatorname{supp}\left[G_{*}^{L}\right]$ in all of the cases in Proposition 7, low types earn no rents in any equilibrium. On the contrary, the rents given up to the high type vary with the form of $\mathbf{G}_{*}$. To handle the three cases in one go, consider the lowest bid of $\operatorname{supp}\left[G_{*}^{H}\right]$, denoted $\underline{B}_{H}$. Since the union of the two bid supports has no gaps, the low type must be indifferent between bidding zero and $\underline{B}_{H}$. This indifference condition provides an alternative expression of $\underline{B}_{H}$. Setting up $u^{W}\left(\underline{B}_{H}, L \mid \mathbf{G}_{*}\right)=0$ from (3) and then solving for $\underline{B}_{H}$ gives us

$$
\underline{B}_{H}=p_{L}(L)\left[V_{L}(L) G_{*}^{L}\left(\underline{B}_{H}\right)+\int_{0}^{\underline{B}_{H}} G_{*}^{L}(x) d x\right] .
$$

We then derive the formula for the information rent of the high type by computing the expected 
payoff $u^{w}\left(\underline{B}_{H}, H \mid \mathbf{G}_{*}\right)$ from (3):

$$
\begin{aligned}
U_{*}^{W}(H) & =-\underline{B}_{H}+p_{H}(L)\left[V_{H}(L) G_{*}^{L}\left(\underline{B}_{H}\right)+\int_{0}^{\underline{B}_{H}} G_{*}^{L}(x) d x\right] \\
& =G_{*}^{L}\left(\underline{B}_{H}\right)\left[\varphi_{H}(L)-\varphi_{L}(L)\right]-\left(p_{L}(L)-p_{H}(L)\right) \int_{0}^{\underline{B}_{H}} G_{*}^{L}(x) d x,
\end{aligned}
$$

where we used (5) to obtain the second expression. This expression holds for all three cases of Proposition 7, where the low type's equilibrium bid distribution at $\underline{B}_{H}$ must satisfy

$$
G_{*}^{L}\left(\underline{B}_{H}\right) \begin{cases}=0 & \text { if } \varphi_{H}(L)<\varphi_{L}(L) \\ \in(0,1) & \text { if } \varphi_{H}(L) \in\left[\varphi_{L}(L), \lambda \varphi_{L}(L)\right) \\ =1 & \text { if } \varphi_{H}(L) \geq \lambda \varphi_{L}(L) .\end{cases}
$$

The bidder's rent in the all-pay auction is given in Proposition 2. Translated to our current two-bidder notation, the rent of the high type can be written

$$
U_{*}^{A}(H)=\max \left\{0, \varphi_{H}(L)-\varphi_{L}(L)\right\}
$$

Comparing (6) and (7), we obtain the following result:

Lemma 4. The war of attrition leaves a lower rent to the bidders than the all-pay auction. This holds strictly whenever the rent in the all-pay auction is non-zero.

Combining Lemmas 3 and 4, we have:

Corollary 2. The war of attrition leaves a strictly lower rent to the bidders than the standard auctions.

We next compare the probability of misallocation between the two all-pay auctions. For this purpose, we investigate the equilibrium path in the $\left(G_{*}^{L}(b), G_{*}^{H}(b)\right)$ phase plane as a function of $b \in[0, \infty)$. The curve marked as WOA in Figure 4 shows the equilibrium path of the war of attrition in the case (iii) of Proposition 7 (the parameters are the same as those used in Figures 2 and 3), that is, when the two bid supports are fully overlapped.

By subtracting the two equations of (DE-2), it can be shown that the low-type bidders drop out from the game with a higher hazard rate in equilibrium. This in turn implies that the ratio of the winning probabilities against the two bidder types, $G_{*}^{H}(b) / G_{*}^{L}(b)$, is increasing in $b$. As a result, the equilibrium path $\left(G_{*}^{L}(b), G_{*}^{H}(b)\right)$ bends upwards until the parametric bid $b$ reaches $\bar{B}_{L}$, where $G_{*}^{L}\left(\bar{B}_{L}\right)=1$. Figure 4 also displays the corresponding path $\left(F_{*}^{L}(b), F_{*}^{H}(b)\right)$, marked as APA, in the all-pay auction with the same parameter values. This curve has the same slope at the origin, but as argued in the previous section, it is actually linear.

Consider next the probability of misallocation. Conditional on one bidder having a low signal and one having a high signal, we are interested in the probability of the low type winning the 


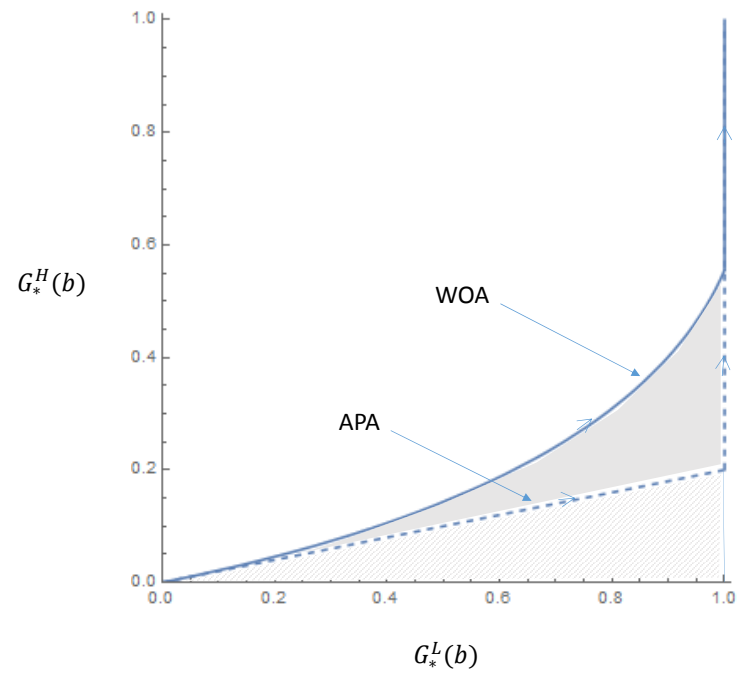

Figure 4: Probability of misallocation: war of attrition vs. all-pay auction

auction. Using the change of variables, the probability can be written as:

$$
\int_{0}^{\bar{B}_{L}} G_{*}^{H}(b) \frac{d G_{*}^{L}(b)}{d b} d b=\int_{0}^{1} G_{*}^{H}(\beta) d \beta
$$

which is just the area under the equilibrium path illustrated by shaded regions in Figure 4 . The probability of misallocation in the case of the all-pay auction is given by the light shaded area while in the war of attrition it is given by the sum of the two shaded areas. We can hence conclude that the probability of misallocation is higher in the case of the war of attrition and we have an unambiguous ranking of the auction formats in terms of their efficiency:

Lemma 5. The probability of an inefficient allocation is higher in the war of attrition than in the all-pay auction. Consequently, we have

$$
L_{*}^{W} \geq L_{*}^{A}
$$

The inequality is strict whenever the probability of misallocation is non-zero in the war of attrition.

Proof. See Appendix A.4.

With the series of lemmas above in hand, we can compare the expected revenue from the war of attrition to that from the other auction formats. Recall that the revenue from auction $\Gamma \in\{A, W, S\}$ can be decomposed as

$$
\Pi_{*}^{\Gamma}=\bar{\Pi}-L_{*}^{\Gamma}-U_{*}^{\Gamma},
$$

where the superscript $\Gamma$ refers to the all-pay auction (A), war of attrition (W), or standard first- or second-price auctions (S), respectively. We start by comparing the war of attrition to the standard 
auctions. Note that the standard auctions are always efficient, so that $L_{*}^{S}=0$. Therefore, the revenue comparison boils down to the comparison between the rent in the standard auction, $U_{*}^{S}$, and the sum of bidder rent and inefficiency loss in the war of attrition, $U_{*}^{W}+L_{*}^{W}$. We show in the appendix that the former always outweighs the latter, and thus the war of attrition is revenuesuperior to the standard auctions.

Proposition 8. The war of attrition raises a higher expected revenue than the standard auctions.

Proof. See Appendix A.5.

We now move to the comparison between the war of attrition and the all-pay auction. Let us first consider the special case of common values, where $V_{H}(L)=V_{L}(H)$ so that the ex post value of the object is the same to every bidder. Then the allocation is always efficient and the rent comparison boils down to comparing the efficiency loss between the auction formats. Lemma 5 above gives $L_{*}^{W} \geq L_{*}^{A}$, so we have immediately:

Proposition 9. In the mineral-rights model (i.e. when $V_{H}(L)=V_{L}(H)$ ), the symmetric equilibrium of the war of attrition generates a higher expected revenue than the symmetric equilibrium of the all-pay auction.

When $V_{H}(L)>V_{L}(H)$, we have to take into account the surplus loss from allocative inefficiency as well as information rents. Note that by Lemma 4 the information rent aspect favors the war of attrition, while by Lemma 5 the efficiency aspect favors the all-pay auction. If $\varphi_{H}(L)-\varphi_{L}(L) \leq 0$, then both auction formats dissipate all the bidder rents, so that the more severe inefficiency of the war of attrition implies higher revenues for the all-pay auction. If $\varphi_{H}(L)-\lambda \varphi_{L}(L) \geq 0$, then both auction formats have a monotonic equilibrium with no allocative inefficiencies, and hence the more effective rent dissipation by the war of attrition generates higher revenue. Note that this latter finding is in full accord with the revenue ranking results in Krishna and Morgan (1997).

There is also a range of parameters $\varphi_{H}(L) \in\left[\varphi_{L}(L), \lambda \varphi_{L}(L)\right)$ where the war of attrition involves overlap in bidding supports but the symmetric equilibrium of the all-pay auction is efficient. In this range, allocative efficiency favors the all-pay auction, whereas the rent for the high type favors the war of attrition. At one end of the parameter range (where $\varphi_{H}(L)-\varphi_{L}(L)$ is positive but close to zero), rents in both mechanisms vanish, and hence the inefficiency of the war of attrition dominates revenue comparison. At the other end of the parameter range (where $\varphi_{H}(L)$ approaches $\lambda \varphi_{L}(L)$ from the left), the inefficiency of the war of attrition disappears and the bidder rent difference relative to the all-pay auction dominates.

Proposition 10. If $V_{H}(L)>V_{L}(H)$, the revenue ranking between the symmetric equilibria of the war of attrition and the all-pay auction is ambiguous. In particular, the all-pay auction generates a strictly higher expected revenue than the war of attrition when $\phi_{H}(L)-\phi_{L}(L) \leq 0$, whereas the war of attrition generates a strictly higher expected revenue than the all pay auction when $\phi_{H}(L)-\lambda \phi_{L}(L) \geq 0$.

Proof. See Appendix A.6. 


\section{Conclusions}

Correlation in signals causes problems for the existence of monotone equilibria in all-pay auctions. This limits seriously the scope of the traditional analysis based on auction theoretic arguments. In a simple model with two types of bidders, we show that the non-existence of monotone equilibria has significant implications for the efficiency of allocations. We show that even if we let the number of players be arbitrarily large, the allocation may be inefficient when the bidders have affiliated private values.

We hope our findings in this simple setting inspires further work in related models. In addition to exploring richer informational models, further research should address contests with multiple prizes as well as contests with less extreme outcome functions and study efficiency and information aggregation in such environments.

\section{A. Omitted Proofs}

\section{A.1. Proof of Lemma 2}

To show that $\psi(n)=V_{H}(n) p_{H}(n)-V_{L}(n) p_{L}(n)$ is single-crossing, it is sufficient to establish the log-supermodularity of $V_{k}(n) p_{k}(n)$ in $k$ and $n$. We know that $p_{k}(n)$ is log-supermodular by the monotone likelihood ratio property. Hence we need to show only that $V_{k}(n)$ is log-supermodular. Since

$$
V_{k}(n)=\mathbb{E}_{\theta}\left[v\left(\theta, t_{i}\right) \mid Y_{i}=n, t_{i}=k\right],
$$

and since we have assumed the log-supermodularity of both $v\left(\theta, t_{i}\right)$ and $p(\theta, \mathbf{t})$, the result follows from the fact that log-supermodularity is preserved by integration and multiplication (See Karlin and Rinott (1980)).

\section{A.2. Proof of Proposition 2}

We shall prove this result through a series of lemmas. The first lemma shows that in any symmetric equilibrium the low-type bidder earns a payoff of zero. This result follows from the fact that the function $\psi$ takes a positive value at $N-1$.

Lemma A.1. In any symmetric equilibrium $\mathbf{F}_{*}, 0 \in \operatorname{supp}\left[F_{*}^{L}\right]$ and as a consequence the low-type bidders earn no rents.

Proof. Suppose to the contrary that $\underline{B}_{L} \equiv \min \left(\operatorname{supp}\left[F_{*}^{L}\right]\right)>0$. Then by Lemma 1 , we must have $0 \in \operatorname{supp}\left[F_{*}^{H}\right]$ and $F_{*}^{H}\left(\underline{B}_{L}\right)>0$. Since the high type is then indifferent between bidding zero and $\underline{B}_{L}$, we can derive an alternative expression of $\underline{B}_{L}$ as follows:

$$
u^{A}\left(\underline{B}_{L}, H \mid \mathbf{F}_{*}\right)=0 \quad \Longrightarrow \quad \underline{B}_{L}=V_{H}(N-1) p_{H}(N-1)\left(F_{*}^{H}\left(\underline{B}_{L}\right)\right)^{N-1} \text {, }
$$


where we used $F_{*}^{L}\left(\underline{B}_{L}\right)=0$. Using this expression, we compute the expected payoff from bidding $\underline{B}_{L}$ to the low type, to obtain

$$
u^{A}\left(\underline{B}_{L}, L \mid \mathbf{F}_{*}\right)=-\underline{B}_{L}+V_{L}(N-1) p_{L}(N-1)\left(F_{*}^{H}\left(\underline{B}_{L}\right)\right)^{N-1}=-\psi(N-1)\left(F_{*}^{H}\left(\underline{B}_{L}\right)\right)^{N-1},
$$

The last expression is strictly negative because $\psi(N-1)>0$ and $F_{*}^{H}\left(\underline{B}_{L}\right)>0$, leading us to a contradiction of $\underline{B}_{L} \in \operatorname{supp}\left[F_{*}^{L}\right]$.

The next lemma presents a sufficient condition for existence of a monotone strategy equilibrium.

Lemma A.2. If $\psi(0) \geq 0$, then the all-pay auction has a unique symmetric BNE in monotone strategies.

Proof. Since $\psi(n)$ is single-crossing, $\psi(0) \geq 0$ implies $\psi(n)>0$ for all $n=1, \cdots, N-1$. Hence the condition implies that an increment in bid is more beneficial to the high type:

$$
\frac{\partial}{\partial b} u^{A}\left(b, H \mid F_{*}\right)-\frac{\partial}{\partial b} u^{A}\left(b, L \mid \mathbf{F}_{*}\right)=\frac{\partial}{\partial b} \sum_{n=0}^{N-1} \psi(n)\left(F_{*}^{H}(b)\right)^{n}\left(F_{*}^{L}(b)\right)^{N-n-1}>0 .
$$

In other words, the bidder's expected payoff function is supermodular in $\left(b ; t_{i}\right)$, so any symmetric equilibrium must be in monotone strategies. ${ }^{15}$ Since $0 \in \operatorname{supp}\left[F_{*}^{L}\right]$ by the previous lemma, and since the low type has a chance to win only if his opponents are all low types in this monotonic equilibrium, we have $\operatorname{supp}\left[F_{*}^{L}\right]=\left[0, \bar{B}_{L}\right]$ where $\bar{B}_{L}:=\max \left(\operatorname{supp}\left[F_{*}^{L}\right]\right)=\underline{B}_{H}:=$ $\min \left(\operatorname{supp}\left[F_{*}^{H}\right]\right)=V_{L}(0) p_{L}(0)$. Furthermore, we can characterize $F_{*}^{L}$ from the indifference condition between bidding zero and every $b \in \operatorname{supp}\left[F_{*}^{L}\right]$ to the low type:

$$
b=V_{L}(0) p_{L}(0)\left(F_{*}^{L}(b)\right)^{N-1}
$$

for each $b \in\left[0, V_{L}(0) p_{L}(0)\right]$. The bid distribution of the low type is therefore uniquely determined.

For the high-type bidders, indifference at all $b \in \operatorname{supp}\left[F_{*}^{H}\right]$ holds if and only if

$$
\sum_{n=0}^{N-1} p_{H}(n) V_{H}(n)\left(F_{*}^{H}(b)\right)^{n}-b=V_{H}(0) p_{H}(0)-V_{L}(0) p_{L}(0),
$$

where the expression on the right-hand side indicates the expected payoff to the high type from bidding $\underline{B}_{H}$, namely his information rent: $\psi(0) \geq 0$. From this indifference condition, we see the

\footnotetext{
${ }^{15} \mathrm{As} u\left(b, k \mid \mathbf{F}_{*}\right)$ is supermodular, we have $\operatorname{supp}\left[F_{*}^{L}\right] \leq \operatorname{supp}\left[F_{*}^{H}\right]$ in the strong set order (See Milgrom and Shannon (1994)). This result has two implications on the equilibrium support. First, each support must be a connected interval, for otherwise there exists a pair of bids $b_{H}<b_{L}$ such that $b_{L} \in \operatorname{supp}\left[F_{*}^{L}\right]$ and $b_{H} \in \operatorname{supp}\left[F_{*}^{H}\right]$. Second, the supports must be disjoint, because the strict supermodularity implies that if two bids, say $b_{1}$ and $b_{2}>b_{1}$, are indifferent to the low type, then $b_{2}$ must be strictly preferred to $b_{1}$ by the high type.
} 
uniqueness of $F_{*}^{H}(b)$ for $b \in \operatorname{supp}\left[F_{*}^{H}\right]$, and using $F_{*}^{H}\left(\bar{B}_{H}\right)=1$, we get

$$
\bar{B}_{H}:=\max \left(\operatorname{supp}\left[F_{*}^{H}\right]\right)=\sum_{n=0}^{N-1} p_{H}(n) V_{H}(n)-V_{L}(0) p_{L}(0) \text {. }
$$

We next examine the case of $\psi(0)<0$. The following lemma demonstrates that in this case the high-type bidders also earn zero rent in equilibrium. Together with Lemma A.1 and A.2, the lemma tells us that there is full rent dissipation if and only if $\psi(0)<0$.

Lemma A.3. If $\psi(0)<0$, then $0 \in \operatorname{supp}\left[F_{*}^{H}\right]$ in every symmetric equilibrium $\mathbf{F}_{*}$, and as a result, the high-type bidders earn a payoff of zero.

Proof. Suppose $\min \left(\operatorname{supp}\left[F_{*}^{H}\right]\right)=\underline{B}_{H}>0$. Since the union of the supports is a connected interval by Lemma 1 , we must have $F_{*}^{L}\left(\underline{B}_{H}\right)>0$. Also, since the low type earns a payoff of zero from bidding $\underline{B}_{H}$ by Lemma A.1, we have $\underline{B}_{H}=V_{L}(0) p_{L}(0)\left(F_{*}^{L}\left(\underline{B}_{H}\right)\right)^{N-1}$. Using this expression, we can compute the corresponding payoff to the high type:

$$
u^{A}\left(\underline{B}_{H}, H \mid \mathbf{F}_{*}\right)=-\underline{B}_{H}+V_{H}(0) p_{H}(0)\left(F_{*}^{L}\left(\underline{B}_{H}\right)\right)^{N-1}=\psi(0)\left(F_{*}^{L}\left(\underline{B}_{H}\right)\right)^{N-1}<0 .
$$

Because every bidder has an option of bidding zero, any bids in the support must yield a nonnegative expected payoff. Hence $\psi(0)<0$ results in $\underline{B}_{H}=0$.

To fully characterize the equilibrium supports, we show in next lemma that each support is a connected interval and that $\operatorname{supp}\left[F_{*}^{L}\right] \subset \operatorname{supp}\left[F_{*}^{H}\right]$.

Lemma A.4. Suppose $\psi(0)<0$. In any symmetric BNE $\mathbf{F}_{*}$, both $\operatorname{supp}\left[F_{*}^{L}\right]$ and $\operatorname{supp}\left[F_{*}^{H}\right]$ are connected intervals.

Proof. Suppose to the contrary that there is an open interval $\left(b_{1}^{\prime}, b_{2}^{\prime}\right) \subset\left[0, \bar{B}_{L}\right]$ such that supp $\left[F_{*}^{L}\right] \cap$ $\left(b_{1}^{\prime}, b_{2}^{\prime}\right)=\varnothing$. Let $\left(b_{1}, b_{2}\right)$ be the maximal (in the sense of set inclusion) open interval such that

$$
\left(b_{1}^{\prime}, b_{2}^{\prime}\right) \subset\left(b_{1}, b_{2}\right) \text { and } \operatorname{supp}\left[F_{*}^{L}\right] \cap\left(b_{1}, b_{2}\right)=\varnothing
$$

Then $b_{1}$ and $b_{2}$ must belong to $\operatorname{supp}\left[F_{*}^{L}\right] \cap \operatorname{supp}\left[F_{*}^{H}\right]$, and $F_{*}^{H}\left(b_{2}\right)>F_{*}^{H}\left(b_{1}\right)$ by Lemma 1 . Using Lemma A.1, we first obtain an alternative expression for $b_{1}$ and $b_{2}$ :

$$
\begin{aligned}
& b_{1}=\sum_{n=0}^{N-1} V_{L}(n) p_{L}(n)\left(F_{*}^{L}\left(b_{1}\right)\right)^{N-1-n}\left(F_{*}^{H}\left(b_{1}\right)\right)^{n} \\
& b_{2}=\sum_{n=0}^{N-1} V_{L}(n) p_{L}(n)\left(F_{*}^{L}\left(b_{1}\right)\right)^{N-1-n}\left(F_{*}^{H}\left(b_{2}\right)\right)^{n},
\end{aligned}
$$


where we used $F_{*}^{L}\left(b_{1}\right)=F_{*}^{L}\left(b_{2}\right)$ to derive the expression of $b_{2}$. Also, it follows from Lemma A.3 that $b_{1} \in \operatorname{supp}\left[F_{*}^{H}\right]$ yields a payoff of zero to the high type, thereby

$$
\begin{aligned}
u^{A}\left(b_{1}, H \mid \mathbf{F}_{*}\right) & =-b_{1}+\sum_{n=0}^{N-1} V_{H}(n) p_{H}(n)\left(F_{*}^{L}\left(b_{1}\right)\right)^{N-1-n}\left(F_{*}^{H}\left(b_{1}\right)\right)^{n} \\
& =\sum_{n=0}^{N-1} \psi(n)\left(F_{*}^{L}\left(b_{1}\right)\right)^{N-1-n}\left(F_{*}^{H}\left(b_{1}\right)\right)^{n}=0 .
\end{aligned}
$$

Observe that the expression $\psi(n)\left(F_{*}^{L}\left(b_{1}\right)\right)^{N-1-n}\left(F_{*}^{H}\left(b_{1}\right)\right)^{n}$ is single-crossing in $n$ as $\psi(n)$ is the unique sign-changing factor in that expression. This implies that

$$
\begin{aligned}
u^{A}\left(b_{2}, H \mid \mathbf{F}_{*}\right) & =\sum_{n=0}^{N-1} \psi(n)\left(F_{*}^{L}\left(b_{1}\right)\right)^{N-1-n}\left(F_{*}^{H}\left(b_{2}\right)\right)^{n} \\
& =\sum_{n=0}^{N-1} \psi(n)\left(F_{*}^{L}\left(b_{1}\right)\right)^{N-1-n}\left(F_{*}^{H}\left(b_{1}\right)\right)^{n} \cdot\left(\frac{F_{*}^{H}\left(b_{2}\right)}{F_{*}^{H}\left(b_{1}\right)}\right)^{n}>0,
\end{aligned}
$$

where the inequality comes from the (discrete version) Folk single-crossing lemma and the fact that $\left(\frac{F_{*}^{H}\left(b_{2}\right)}{F_{*}^{H}\left(b_{1}\right)}\right)^{n}$ is a strictly increasing function in $n$. Therefore, the high type is strictly better off by bidding $b_{2}$ rather than $b_{1}$, contradicting the indifference condition between $b_{1}$ and $b_{2}$ to the high type. A similar argument can be used to establish that $\operatorname{supp}\left[F_{*}^{H}\right]$ is also connected.

The proof of existence and uniqueness of equilibrium for the case $\psi(0)<0$ is based on the following lemma:

Lemma A.5. Define a function $G:[0,1] \times[0,1] \rightarrow \Re$ as

$$
G(x, y)=\sum_{n=0}^{N-1} g(n) x^{n} y^{N-n-1},
$$

where the function $g:\{0,1, \cdots, N-1\} \rightarrow \Re$ is single-crossing, $g(0)<0$, and $\sum_{n=0}^{N-1} g(n)>0$. Then there exists a unique mapping $\xi:(0,1] \rightarrow(0,1)$ such that $G(\xi(y), y)=0$ for every $y \in(0,1]$. Furthermore, the mapping $\xi$ is continuous, strictly increasing, and $\lim _{y \downarrow} \xi(y)=0$.

Proof. We begin by enumerating some properties of function $G$. First, $G$ is clearly continuous, and it is easy to check that for any $x>0$ there is some $\delta_{x}>0$ such that $G(x, y)>0$ for $y \in\left(0, \delta_{x}\right)$, and for any $y>0$ there is some $\delta_{y}>0$ such that $G(x, y)<0$ for $x \in\left(0, \delta_{y}\right)$. In particular, $G(1, y)>0$ for every $y \in(0,1)$. This follows from the fact that $g(n)$ is single crossing in $n$, that $\sum_{n=1}^{N-1} g(n)>0$, and that $y^{N-n-1}$ is strictly increasing in $n$ for $y \in(0,1)$. Consequently, there exists a pair of $(x, y) \in(0,1) \times(0,1)$ at which $G(x, y)=0$.

The following pairwise strict single-crossing property of $G$ is the key to the lemma: if $G(x, y)=$ 0 for some $(x, y)$, then

$$
G\left(x^{\prime}, y\right)<0 \text { for } x^{\prime} \in(0, x) \text { but } G\left(x^{\prime}, y\right)>0 \text { for } x^{\prime} \in(x, 1)
$$




$$
G\left(x, y^{\prime}\right)>0 \text { for } y^{\prime} \in(0, y) \text { but } G\left(x, y^{\prime}\right)<0 \text { for } y^{\prime} \in(y, 1)
$$

To prove (A.1), fix $x \in(0,1)$ and $y \in(0,1)$ such that $G(x, y)=\sum_{n=0}^{N-1} g(n) x^{n} y^{N-n-1}=0$. Then for any $x^{\prime} \neq x$, we have

$$
G\left(x^{\prime}, y\right)=\sum_{n=0}^{N-1} g(n)\left(x^{\prime}\right)^{n} y^{N-n-1}=\sum_{n=0}^{N-1} g(n) x^{n} y^{N-n-1}\left(\frac{x^{\prime}}{x}\right)^{n} .
$$

Since $g(n)$ is single-crossing in $n$, so is $g(n) y^{N-n-1} x^{n}$ because both $y^{N-n-1}$ and $x^{n}$ are signpreserving functions of $n$. For $x^{\prime}>(<) x$, the fraction $\left(\frac{x^{\prime}}{x}\right)^{n}$ is strictly increasing (decreasing, respectively) in $n$. Consequently, (A.1) follows by the Folk single-crossing lemma. The proof for (A.2) is completely analogous so is omitted.

Using the properties of $G$ just established, we can now prove the lemma. Fix $y \in(0,1]$. Since $G(x, y)<0$ for $x$ sufficiently small and $G(1, y)>0$, there is some $x$ such that $G(x, y)=0$ by continuity of $G$. Because $G(x, y)$ as a function of $x$ satisfies the single-crossing property by (A.1), this sign-changing point is unique, and hence defines a unique $\xi(y)$ for which $G(\xi(y), y)=0$.

To see that $\lim _{y \downarrow} \xi(y)=0$, recall that for every $x>0$, there exists a $\delta_{x}>0$ such that $G(x, y)>$ 0 for all $y \in\left(0, \delta_{x}\right)$. This means that for all $y<\delta_{x}$, we have $\xi(y)<x$. Since $x$ can be chosen arbitrarily low, it follows that $\lim _{y \downarrow} \xi(y)=0$. Finally, continuity and strict monotonicity of $\xi(y)$ follow in a straightforward manner from the continuity and pairwise strict single-crossing property of $G(x, y)$. The proof is now complete.

The existence and uniqueness of equilibrium can be established as follows.

Proof. By Lemmas A.1, A.3 and A.4, when $\psi(0)<0$, in any equilibrium we must have $0 \in$ $\operatorname{supp}\left[F_{*}^{L}\right] \cap \operatorname{supp}\left[F_{*}^{H}\right]$ and both supports are connected intervals. Consequently, there must be some interval $\left[0, \bar{B}_{L}\right]$ where the two supports overlap. Note that every bid $b \in\left[0, \bar{B}_{L}\right]$ must yield zero expected payoff (i.e., the same payoff) to both types. Below we demonstrate that there exists only one pair of $\left(F_{*}^{L}, F_{*}^{H}\right)$ satisfying this property.

For each $y \in(0,1]$, we define $\xi:(0,1] \rightarrow(0,1)$ as the solution to the equation:

$$
\sum_{n=0}^{N-1}[\underbrace{V_{H}(n) p_{H}(n)-V_{L}(n) p_{L}(n)}_{=\psi(n)}](\xi(y))^{n} y^{N-n-1}=0 .
$$

As the function $\psi(n)$ satisfies all the given properties for function $g$ in Lemma A.5, we know from that lemma that there exists a unique continuous and strictly increasing mapping satisfying (A.3) and $\lim _{y \rightarrow 0} \xi(y)=0$.

Given this function $\xi$, we define $\bar{B}_{L}$ as

$$
\sum_{n=0}^{N-1} V_{H}(n) p_{H}(n)(\xi(1))^{n}=\bar{B}_{L}
$$


For each $b<\bar{B}_{L}$, let $F_{*}^{L}(b) \in[0,1)$ denote the unique value of $y$ that solves the equation

$$
\sum_{n=0}^{N-1} V_{H}(n) p_{H}(n)(\xi(y))^{n} y^{N-n-1}=b .
$$

As the expression on the left-hand side of the equation is strictly increasing in $y$ and $b<\bar{B}_{L}$, the solution $F_{*}^{L}(b)$ exists and is unique. Furthermore, it is easy to check that the solution $F_{*}^{L}(b)$ retains all the necessary properties of a distribution function: it is strictly increasing and continuous in $b, F_{*}^{L}(0)=0$, and $F_{*}^{L}\left(\bar{B}_{L}\right)=1$ (by definition of $\bar{B}_{L}$ ). Label $\xi\left(F_{*}^{L}(b)\right)=F_{*}^{H}(b)$. Then $F_{*}^{H}(b)$ is strictly increasing in $b$ and $F_{*}^{H}(0)=0$. This process characterizes the symmetric equilibrium on $\operatorname{supp}\left[F_{*}^{L}\right] \cap \operatorname{supp}\left[F_{*}^{H}\right]$.

To characterize the equilibrium on $\operatorname{supp}\left[F_{*}^{H}\right] \cap\left\{\operatorname{supp}\left[F_{*}^{L}\right]\right\}^{c}$, let

$$
\bar{B}_{H}=\sum_{n=1}^{N-1} V_{H}(n) p_{H}(n) \text {. }
$$

For each $b \in\left(\bar{B}_{L}, \bar{B}_{H}\right)$, set $F_{*}^{L}(b)=1$ and define $F_{*}^{H}(b)$ as the solution to the equation

$$
\sum_{n=0}^{N-1} V_{H}(n) p_{H}(n) x^{n}=b
$$

Since the left-hand side is strictly increasing in $x, F_{*}^{H}(b)$ is uniquely determined.

\section{A.3. Proof of Proposition 7}

We analyze the system of linear differential equations (DE-2). For each type $k=L, H$, we define $G^{k}(b):=1-G_{*}^{k}(b)$ and $g^{k}(b):=-g_{*}^{k}(b)$. This notation is for convenience since it allows us to rewrite (DE-2) as a homogeneous system:

$$
\left[\begin{array}{l}
g^{L}(b) \\
g^{H}(b)
\end{array}\right]=h\left[\begin{array}{ll}
a_{1} & a_{2} \\
a_{3} & a_{4}
\end{array}\right]\left[\begin{array}{l}
G^{L}(b) \\
G^{H}(b)
\end{array}\right]:=A\left[\begin{array}{l}
G^{L}(b) \\
G^{H}(b)
\end{array}\right]
$$

where

$$
\begin{aligned}
h & :=-\left[\varphi_{L}(L) \varphi_{H}(H)-\varphi_{L}(H) \varphi_{H}(L)\right]^{-1}<0 \\
a_{1} & :=\varphi_{H}(H) p_{L}(L)-\varphi_{L}(H) p_{H}(L)>0 \\
a_{2} & :=\varphi_{H}(H) p_{L}(H)-\varphi_{L}(H) p_{H}(H)=p_{L}(H) p_{H}(H)\left[V_{H}(H)-V_{L}(H)\right]>0 \\
a_{3} & :=-\varphi_{H}(L) p_{L}(L)+\varphi_{L}(L) p_{H}(L)=p_{L}(L) p_{H}(L)\left[V_{L}(L)-V_{H}(L)\right]<0 \\
a_{4} & :=-\varphi_{H}(L) p_{L}(H)+\varphi_{L}(L) p_{H}(H) .
\end{aligned}
$$

Unlike the sign of the other parameters, the sign of $a_{4}$ is undetermined by the primitives of the 
model, but as will be seen, whenever the two bid supports are overlapped in equilibrium, we have $a_{4}>0$. The desired symmetric equilibrium can be characterized by a straightforward phase-plane analysis of (DE-1) and (A.4) over the plane $\left[G^{L}(b), G^{H}(b)\right] \in[0,1] \times[0,1]$.

We start by defining the region in the plane where overlapping bidding supports are feasible, i.e. the region where (A.4) admits $g^{L}(b) \leq 0$ and $g^{H}(b) \leq 0$ (recall that $g^{k}(b):=-g_{*}^{k}(b)$ ). Since $a_{1}>0, a_{2}>0$ and $h<0$, we see from (A.4) that $g^{L}(b) \leq 0$ holds always. For $g^{H}(b) \leq 0$, on the other hand, we need $a_{4}>0$ and $a_{3} G^{L}(b)+a_{4} G^{H}(b) \geq 0$, which outlines the region $\mathcal{D}$ where overlapping supports are feasible:

$$
\mathcal{D}:=\left\{\left[G^{L}, G^{H}\right] \in[0,1] \times[0,1]: \frac{G^{H}}{G^{L}} \geq-\frac{a_{3}}{a_{4}}\right\} .
$$

To derive explicit solutions for the bid distributions, we note that the matrix $A$ has two real eigenvalues

$$
r_{1}=\frac{h}{2}\left(a_{1}+a_{4}-\bar{a}\right)<0 \text { and } r_{2}=\frac{h}{2}\left(a_{1}+a_{4}+\bar{a}\right)<r_{1},
$$

where we introduce another parameter $\bar{a}:=\sqrt{\left(a_{1}-a_{4}\right)^{2}+4 a_{2} a_{3}}$ for a simple exposition. To see that both $r_{1}$ and $r_{2}$ are real-valued, write

$$
\begin{aligned}
a_{1}-a_{4} & =p_{L}(L) p_{H}(H)\left\{V_{H}(H)-V_{L}(L)\right\}+p_{L}(H) p_{H}(L)\left\{V_{H}(L)-V_{L}(H)\right\} \\
& \geq p_{L}(L) p_{H}(H)\left\{V_{H}(H)-V_{L}(H)\right\}+p_{L}(H) p_{H}(L)\left\{V_{H}(L)-V_{L}(L)\right\},
\end{aligned}
$$

where the inequality follows from $V_{L}(H) \geq V_{L}(L)$ and log-supermodularity of $p$. As a result,

$$
\left(a_{1}-a_{4}\right)^{2} \geq\left[p_{L}(L) p_{H}(H)\left\{V_{H}(H)-V_{L}(H)\right\}+p_{L}(H) p_{H}(L)\left\{V_{H}(L)-V_{L}(L)\right\}\right]^{2}
$$

Observe that $-a_{2} a_{3}$ is simply the product of the two terms inside the bracket above. Consequently, we have $\left(a_{1}-a_{4}\right)^{2}+4 a_{2} a_{3} \geq 0$.

The general solution to (A.4) is then

$$
\left[\begin{array}{l}
G^{L}(b) \\
G^{H}(b)
\end{array}\right]=c_{1}\left[\begin{array}{c}
k_{1} \\
1
\end{array}\right] e^{r_{1} b}+c_{2}\left[\begin{array}{c}
k_{2} \\
1
\end{array}\right] e^{r_{2} b}
$$

where $c_{1}$ and $c_{2}$ are free parameters and $k_{1}$ and $k_{2}$ are normalized eigenvector components:

$$
k_{1}=\frac{a_{1}-a_{4}-\bar{a}}{2 a_{3}}<0 \text { and } k_{2}=\frac{a_{1}-a_{4}+\bar{a}}{2 a_{3}}<k_{1} .
$$

We are now ready to analyze the three mutually exclusive cases in Proposition 7. 


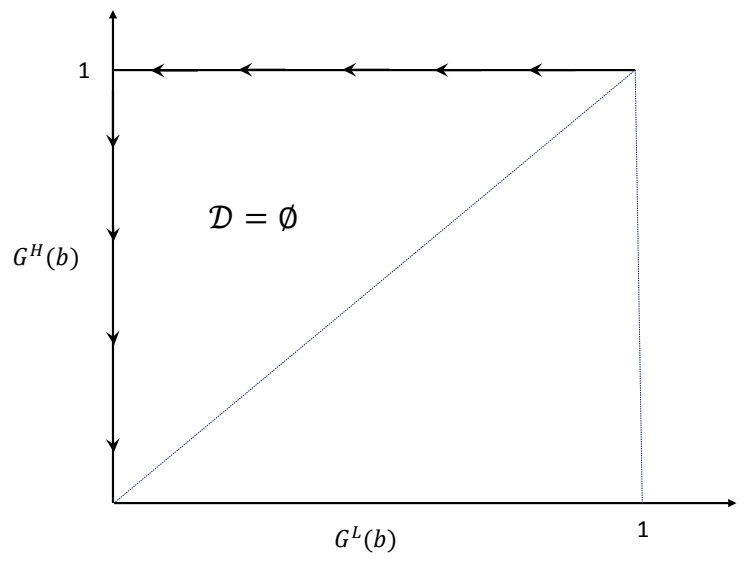

Figure 5: Equilibrium Trajectory in Case (i).

Case (i). $\varphi_{H}(L) \geq \lambda \varphi_{L}(L)$

In this case, $a_{3}<a_{4} \leq 0$ and therefore $\mathcal{D}=\varnothing$, meaning that the system (DE-2) does not have a feasible solution with $\left(g_{*}^{L}(b), g_{*}^{H}(b)\right) \gg 0$. Hence there should be no overlap between the two bid supports. The equilibrium $\left(G_{*}^{L}, G_{*}^{H}\right)$ described in case (i) of Proposition 7 follows by solving the necessary indifference condition (DE-1) separately for $G_{*}^{L}$ and $G_{*}^{H}$. First, (DE-1) for type $k=L$,

$$
\frac{p_{L}(L) g_{*}^{L}(b) V_{L}(L)}{1-p_{L}(L) G_{*}^{L}(b)}=1
$$

with initial condition $G_{*}^{L}(0)=0$ gives the formula for $G_{*}^{L}(b)$. Then setting the boundary condition $G_{*}^{L}\left(\bar{B}_{L}\right)=1$ (recall that $\bar{B}_{L}=\sup \operatorname{supp}\left[G_{*}^{L}\right]$ ) gives the formula for $\bar{B}_{L}$. Similarly, (DE-1) for type $k=H$ is

$$
\frac{p_{H}(H) g_{*}^{H}(b) V_{H}(H)}{1-p_{L}(L)-p_{H}(H) G_{*}^{H}(b)}=1,
$$

which with initial condition $G_{*}^{H}\left(\bar{B}_{L}\right)=0$ gives the formula for $G_{*}^{H}(b)$. Figure 5 displays the equilibrium trajectory which is marked by arrows (recall that $G^{L}(b):=1-G_{*}^{L}(b)$ and $G^{H}(b):=$ $\left.1-G_{*}^{H}(b)\right)$.

Case (ii). $\varphi_{L}(L) \leq \varphi_{H}(L)<\lambda \varphi_{L}(L)$

We now work directly with functions $\left(G^{L}, G^{H}\right)$. In Case (ii), we have $a_{4}>0$ and

$$
\left[G^{L}(b), G^{H}(b)\right] \in \mathcal{D} \Leftrightarrow \frac{G^{H}(b)}{G^{L}(b)}>-\frac{a_{3}}{a_{4}}=\frac{\varphi_{H}(L) p_{L}(L)-\varphi_{L}(L) p_{H}(L)}{\varphi_{L}(L) p_{H}(H)-\varphi_{H}(L) p_{L}(H)}>1 .
$$

Since $G^{H}(0) / G^{L}(0)=1<-a_{3} / a_{4}$, bid zero cannot be in the support of $G_{*}^{H}$. It is easy to check that the only possibility is that there is a bid interval $\left[0, \underline{B}_{H}\right]$, where only type $L$ is active, and $G_{*}^{L}(b)$ in that interval follows from the indifference condition (DE-1) for type $L$ together with 
initial condition $G_{*}^{L}(0)=0$. The formula for $\underline{B}_{H}$ is

$$
\underline{B}_{H}=V_{L}(L) \log \left[\frac{\varphi_{H}(L)-V_{L}(L) p_{H}(L)}{\varphi_{L}(L)-V_{L}(L) p_{H}(L)}\right]<V_{L}(L) \log \left[p_{L}(H)\right]^{-1},
$$

This is derived from the condition

$$
\frac{1}{G^{L}\left(\underline{B}_{H}\right)}=\frac{1}{1-G_{*}^{L}\left(\underline{B}_{H}\right)}=-\frac{a_{3}}{a_{4}}
$$

that defines the point where the equilibrium path hits the region $\mathcal{D}$ (see Figure 6-(a), where the shaded area indicates $\mathcal{D}$ ). It is easy to verify from (A.4) that we have a trajectory $\left[G^{L}(b), G^{H}(b)\right]$ with $g^{L}(b)<g^{H}(b)<0$ for $b \in\left[\underline{B}_{H}, \bar{B}_{L}\right]$, where $\bar{B}_{L}$ is pinned down by $G^{L}\left(\bar{B}_{L}\right)=0$. To get an explicit formula for the bidding distributions, we plug initial conditions $G^{L}\left(\underline{B}_{H}\right)=-\frac{a_{4}}{a_{3}}$ and $G^{H}\left(\underline{B}_{H}\right)=1$ into (A.5), which gives:

$$
\begin{aligned}
G^{L}(b) & =\widetilde{c}_{1} \exp \left[r_{1}\left(b-\underline{B}_{H}\right)\right]+\widetilde{c}_{2} \exp \left[r_{2}\left(b-\underline{B}_{H}\right)\right], \\
G^{H}(b) & =\widetilde{d}_{1} \exp \left[r_{1}\left(b-\underline{B}_{H}\right)\right]+\widetilde{d}_{2} \exp \left[r_{2}\left(b-\underline{B}_{H}\right)\right],
\end{aligned}
$$

where

$$
\begin{array}{llrl}
\widetilde{c}_{1} & =\frac{\left(a_{1}+a_{4}+\bar{a}\right)\left(a_{1}-a_{4}-\bar{a}\right)}{4 a_{3} \bar{a}}<0, & \widetilde{c}_{2}=-\frac{\left(a_{1}+a_{4}-\bar{a}\right)\left(a_{1}-a_{4}+\bar{a}\right)}{4 a_{3} \bar{a}}>0 \\
\widetilde{d}_{1}=\frac{a_{1}+a_{4}+\bar{a}}{2 \bar{a}}>0, & \widetilde{d}_{2}=-\frac{a_{1}+a_{4}-\bar{a}}{2 \bar{a}}<0,
\end{array}
$$

and $\bar{B}_{L}$ is obtained from boundary condition $G^{L}\left(\bar{B}_{L}\right)=0$ :

$$
\bar{B}_{L}=\underline{B}_{H}+\left(-\frac{1}{\bar{a} h}\right) \cdot \log \left(\frac{a_{4}\left(a_{1}-a_{4}+\bar{a}\right)-2 a_{2} a_{3}}{a_{4}\left(a_{1}-a_{4}-\bar{a}\right)-2 a_{2} a_{3}}\right) .
$$

The remaining part of $G^{H}(b)$ for $b>\bar{B}_{L}$ follows directly from indifference condition (DE-1) for type $H$, where the initial condition $G^{H}\left(\bar{B}_{L}\right)$ is obtained by computing (A.7) at $b=\bar{B}_{L}$.

Case (iii). $\varphi_{H}(L)<\varphi_{L}(L)$

In the last case, all points $\left[G^{L}(b), G^{H}(b)\right]$ satisfying $0 \leq G^{L}(b) \leq G^{H}(b)$ are in the set $\mathcal{D}$, including the initial point $\left[G^{L}(0), G^{H}(0)\right]=[1,1]$. The system (DE-2) then pins down a unique trajectory from $\left[G^{L}(0), G^{H}(0)\right]=[1,1]$ to $\left[G^{L}\left(\bar{B}_{L}\right), G^{H}\left(\bar{B}_{L}\right)\right]$, where $G^{H}\left(\bar{B}_{L}\right)>0$ and where $\bar{B}_{L}$ defined by $G^{L}\left(\bar{B}_{L}\right)=0$ (see Figure 6-(b)). Using initial conditions $G^{L}(0)=G^{H}(0)=1$, we get the formulas for the bidding distributions from (A.5):

$$
\begin{aligned}
G^{L}(b) & =\widehat{c}_{1} e^{r_{1} b}+\widehat{c}_{2} e^{r_{2} b}, \\
G^{H}(b) & =\widehat{d_{1}} e^{r_{1} b}+\widehat{d_{2}} e^{r_{2} b},
\end{aligned}
$$




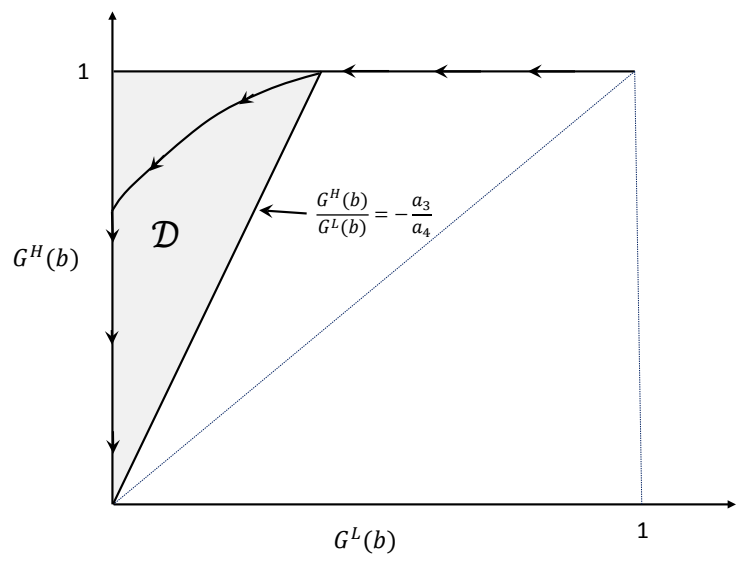

(a) Case (ii)

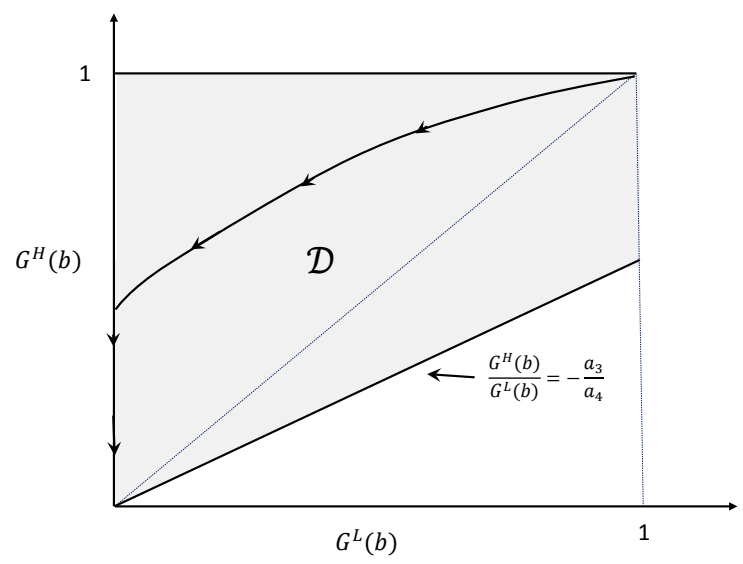

(b) Case (iii)

Figure 6: Equilibrium Trajectory in the war-of-attrition

where

$$
\begin{array}{lll}
\widehat{c}_{1}=\frac{\left(a_{1}-a_{4}-2 a_{3}+\bar{a}\right)\left(a_{1}-a_{4}-\bar{a}\right)}{4 a_{3} \bar{a}}<0, & \widehat{c_{2}}=\frac{\left(-a_{1}+a_{4}+2 a_{3}+\bar{a}\right)\left(a_{1}-a_{4}+\bar{a}\right)}{4 a_{3} \bar{a}}>0, \\
\widehat{d_{1}}=\frac{a_{1}-a_{4}-2 a_{3}+\bar{a}}{2 \bar{a}}>0, & \widehat{d_{2}}=\frac{-a_{1}+a_{4}+2 a_{3}+\bar{a}}{2 \bar{a}}<0,
\end{array}
$$

and the highest bid $\bar{B}_{L}$ in the low-type bid support is determined by $G_{*}^{L}\left(\bar{B}_{L}\right)=0$ as before:

$$
\bar{B}_{L}=\left(-\frac{1}{\bar{a} h}\right) \cdot \log \left(\frac{\left(a_{1}-a_{4}-2 a_{3}-\bar{a}\right)\left(a_{1}-a_{4}+\bar{a}\right)}{\left(a_{1}-a_{4}-2 a_{3}+\bar{a}\right)\left(a_{1}-a_{4}-\bar{a}\right)}\right) .
$$

Lastly, the part $G_{*}^{H}$ for $b>\bar{B}_{L}$ can be obtained from indifference condition (DE-1) for type $k=H$, where the initial condition $G^{H}\left(\bar{B}_{L}\right)$ is obtained by computing (A.9) at $b=\bar{B}_{L}$.

\section{A.4. Proof of Lemma 5}

We compare the probability of misallocation between the war of attrition and the all-pay auction separately for the three cases enumerated in Proposition 7. The first two cases are easy: $L_{*}^{W}=$ $L_{*}^{A}=0$ in case (i) and $L_{*}^{A}=0$ but $L_{*}^{W}>0$ in case (ii).

In Case (iii), the symmetric equilibrium features a full overlap in both auction formats. We computed in the proof of Proposition 7 the equilibrium bid distributions as:

$$
G_{*}^{L}(b)=1-\widehat{c}_{1} e^{r_{1} b}-\widehat{c}_{2} e^{r_{2} b} \text { and } G_{*}^{H}(b)=1-\widehat{d}_{1} e^{r_{1} b}-\widehat{d}_{2} e^{r_{2} b},
$$

where $r_{1}, r_{2}, \widehat{c}_{1}, \widehat{c}_{2}, \widehat{d_{1}}$, and $\widehat{d_{2}}$ are real numbers such that $r_{2}<r_{1}<0, \widehat{c_{1}}<0, \widehat{c}_{2}>0, \widehat{d_{1}}>0$, and 
$\widehat{d_{2}}<0$. The slope of the equilibrium path can then be written as

$$
\frac{d G_{*}^{H}(b)}{d G_{*}^{L}(b)}=\frac{-\widehat{d}_{1} r_{1} e^{r_{1} b}-\widehat{d}_{2} r_{2} e^{r_{2} b}}{-\widehat{c}_{1} r_{1} e^{r_{1} b}-\widehat{c}_{2} r_{2} e^{r_{2} b}}=\frac{\widehat{d_{1}}+\widehat{d}_{2} \frac{r_{2}}{r_{1}}{ }^{\left(r_{2}-r_{1}\right) b}}{\widehat{c}_{1}+\widehat{c}_{2} \frac{r_{2}}{r_{1}} e^{\left(r_{2}-r_{1}\right) b}}
$$

where the last step divides both sides by a positive number $-r_{1} e^{r_{1} b}$ so that both the nominator and denominator remain positive. Given that $\widehat{d_{2}}<0$ and $\widehat{c_{2}}>0$, we see that the nominator is increasing in $b$ while the denominator is decreasing in $b$, so that the slope $\frac{d G_{*}^{H}(b)}{d G_{*}^{L}(b)}$ increases in $b$. In other words, the equilibrium path bends upwards as $b$ increases, as displayed in Figure 4.

The corresponding bidding distributions in the two-player all-pay auction are uniform. Denoting them by $F_{*}^{L}(b)$ and $F_{*}^{H}(b)$, we can write them in terms of the notation used here as $F_{*}^{L}(b)=-\left(r_{1} \widehat{c}_{1}+r_{2} \widehat{c}_{2}\right) b$ and $F_{*}^{H}(b)=-\left(r_{1} \widehat{d}_{1}+r_{2} \widehat{d}_{2}\right) b$. Hence it follows that

$$
\frac{d F_{*}^{H}(b)}{d F_{*}^{L}(b)}=\frac{\widehat{d_{1}} r_{1}+\widehat{d}_{2} r_{2}}{\widehat{c}_{1} r_{1}+\widehat{c}_{2} r_{2}}=\lim _{b \downarrow 0} \frac{d G_{*}^{H}(b)}{d G_{*}^{L}(b)} .
$$

Hence the slopes of the equilibrium paths in the two auction formats coincide at the origin. Therefore the area under the equilibrium path of the war of attrition is strictly larger than that of the all-pay auction, which means that the probability of misallocation is higher in the war of attrition.

\section{A.5. Proof of Proposition 8}

When $\varphi_{H}(L) \geq \varphi_{L}(L) \cdot \lambda$, we know from Proposition 7 that $L_{*}^{W}=0$. Hence the ranking $\Pi_{*}^{W}>\Pi_{*}^{S}$ is immediate from Corollary 2.

When $\varphi_{H}(L) \leq \varphi_{L}(L)$, zero is in the support of both types in the war of attrition. In this case, the mechanism extracts full surplus but suffers from inefficiency. The social cost of the inefficient allocation amounts to $V_{H}(L)-V_{L}(H)$ and can occur only when one of the bidders is of type $H$ and the other is of type $L$, i.e., with probability $\mathbb{P}(1)$. The surplus loss $L_{*}^{W}$ is hence bounded by $\mathbb{P}(1)\left[V_{H}(L)-V_{L}(H)\right]$, which is less than $\mathbb{P}(1)\left[V_{H}(L)-V_{L}(L)\right]$, i.e. the information rent given up to the bidders in the standard auctions. Therefore, we obtain $\Pi_{*}^{W}>\Pi_{*}^{S}$.

In the remaining case, $\varphi_{H}(L) \in\left(\varphi_{L}(L), \varphi_{L}(L) \cdot \lambda\right)$, the war of attrition suffers from an inefficient allocation and at the same time leaves rents to the high type. For revenue comparison, first note that a surplus loss can occur only if the low type makes a bid above $\underline{B}_{H}$. In this case the surplus is reduced by $V_{H}(L)-V_{L}(H)$, which is smaller than $V_{H}(L)-V_{L}(L)$. This gives us an upper bound for $L_{*}^{W}$ :

$$
L_{*}^{W}<\mathbb{P}(1)\left(1-G_{*}^{L}\left(\underline{B}_{H}\right)\right)\left[V_{H}(L)-V_{L}(L)\right] .
$$

Furthermore, we see from (6) that the high-type bidders' rent is bounded from above by $G_{*}^{L}\left(\underline{B}_{H}\right) p_{H}(L)\left[V_{H}(L)-V_{L}(L)\right.$. By summing over the possible realizations of the types, we get a 
bound for the aggregate information rent:

$$
\begin{aligned}
\sum_{n=1}^{2} \mathbb{P}(n) \cdot n \cdot U_{*}^{W}(H) & <\sum_{n=1}^{2} \mathbb{P}(n) \cdot n \cdot G_{*}^{L}\left(\underline{B}_{H}\right) p_{H}(L)\left[V_{H}(L)-V_{L}(L)\right] \\
& =\mathbb{P}(1) G_{*}^{L}\left(\underline{B}_{H}\right)\left[V_{H}(L)-V_{L}(L)\right]
\end{aligned}
$$

where the last equality follows from the fact that $\mathbb{P}(1)=p_{H}(L) \sum_{n=1}^{2} \mathbb{P}(n) \cdot n$. Combining the two upper bounds and using the fact that $F_{*}^{L}\left(\underline{B}_{H}\right) \leq 1$, we obtain:

$$
\Pi_{*}^{W}>\bar{\Pi}-\mathbb{P}(1)\left[V_{H}(L)-V_{L}(L)\right]=\Pi_{*}^{S} .
$$

Therefore, the expected revenue in the war of attrition is higher than in standard auctions in all of the cases.

\section{A.6. Proof of Proposition 10}

When $\varphi_{H}(L) \leq \varphi_{L}(L)$, the bid zero is in the support of both types in both auction formats, and hence the rents are fully dissipated; $U_{*}^{A}(H)=U_{*}^{W}(H)=0$. By Lemma 5, the probability of misallocation is strictly higher in the war of attrition than in the all-pay auction. With $V_{H}(L)>$ $V_{L}(H)$ this implies $L_{*}^{W}>L_{*}^{A}$ and so $\Pi_{*}^{A}>\Pi_{*}^{W}$.

When $\varphi_{H}(L)-\lambda \varphi_{L}(L) \geq 0$, the bidding supports are disjoint and the allocation is efficient in both mechanisms and $L_{*}^{W}=L_{*}^{A}=0$. By Lemma 3 we have $U_{*}^{A}(H)>U_{*}^{W}(H)$, and therefore we obtain $\Pi_{*}^{A}<\Pi_{*}^{W}$.

\section{B. Supplementary material}

Supplementary material related to this article can be found online at https:/ /ars.elscdn.com/content/image/1-s2.0-S0022053118301534-mmc1.pdf.

\section{References}

Amann, E. and LeINinger, W. (1996). Asymmetric All-Pay Auctions with Incomplete Information: The Two-Player Case. Games and Economic Behavior, 14 (1), 1-18.

Baye, M. R., Kovenock, D. and De VRIES, C. G. (1993). Rigging the Lobbying Process: An Application of the All-Pay Auction. American Economic Review, 83 (1), $289-94$.

Che, Y.-K. and Gale, I. L. (1998). Caps on Political Lobbying. American Economic Review, 88 (3), 643-651.

Hillman, A. L. and Riley, J. G. (1989). Politically Contestable Rents And Transfers. Economics and Politics, 1 (1), 17-39. 
KAPLAN, T. and ZAMIR, S. (2015). Advances in auctions. In Handbook of Game Theory with Economic Applications, vol. 4, 7, Elsevier, pp. 381-453.

KARLIN, S. and RINOTT, Y. (1980). Classes of orderings of measures and related correlation inequalities. i. multivariate totally positive distributions. Journal of Multivariate Analysis, 10 (4), 467-498.

Klemperer, P. and Bulow, J. (1999). The Generalized War of Attrition. American Economic Review, 89 (1), 175-189.

KrishnA, V. and Morgan, J. (1997). An Analysis of the War of Attrition and the All-Pay Auction. Journal of Economic Theory, 72 (2), 343-362.

LANDSBERGER, M. (2007). Non-existence of monotone equilibria in games with correlated signals. Journal of Economic Theory, 132 (1), 119-136.

LU, J. and PARreiras, S. O. (2017). Monotone equilibrium of two-bidder all-pay auctions Redux. Games and Economic Behavior, 104 (C), 78-91.

Maskin, E. S. and RILey, J. G. (1985). Auction Theory with Private Values. American Economic Review, 75 (2), 150-155.

Milgrom, P. and SHANNON, C. (1994). Monotone comparative statics. Econometrica, 62 (1), 15780.

Milgrom, P. R. and Weber, R. J. (1982). A theory of auctions and competitive bidding. Econometrica, 50 (5), 1089-1122.

Murto, P. and VÄLIMÄKI, J. (2017). Common Value Auctions with Costly Entry. Tech. rep., Aalto University School of Business and HECER.

Rentschler, L. and TUROCY, T. L. (2016). Two-bidder all-pay auctions with interdependent valuations, including the highly competitive case. Journal of Economic Theory, 163 (C), 435-466.

SIEGEL, R. (2009). All-Pay Contests. Econometrica, 77 (1), 71-92.

- (2010). Asymmetric Contests with Conditional Investments. American Economic Review, 100 (5), 2230-2260.

- (2014). Asymmetric all-pay auctions with interdependent valuations. Journal of Economic Theory, 153 (C), 684-702. 